\title{
List of insect fauna (Insecta) of Kopački Rit Nature Park (NE Croatia)
}

\author{
Kopački Rit Tabiat Parkı (NE Hırvatistan) böcek faunasının (Insecta) listesi
}

\section{Stjepan KRČMAR ${ }^{1}$}

\begin{abstract}
Summary
In the Kopački Rit Nature Park, 895 species of insects have been identified, and classified into 8 orders, 13 suborders and 90 families. Only two species from the order Coleoptera belong to the higher threat category as Vulnerable (VU) according to IUCN Red List criteria, whereas 8 species are listed on Annex II of the Bern Convention or Annexes II and/or IV of the EU Habitats Directive. 118 species of insects classified into 35 families and 6 orders are added to the Fauna Europaea database. Synonyms for 90 species of insects classified into 7 orders are updated according to recent data from the Fauna Europaea database.
\end{abstract}

Key words: Insecta, Kopački Rit, Croatia, Europe

\section{Özet}

Kopački Rit Nature Park'ta 8 takım, 13 alttakım ve 90 familyadan 895 böcek türü teşhis edilmiştir. IUCN kırmızı liste kriterlerine göre yüksek tehdit kategorisinde Coleoptera takımına ait sadece iki tür hassas tür (VU) olarak belirlenmiş, 8 tür ise Dördüncü $A B$ Habitat Direktifi ve/vaya Bern Sözleşmesi Ek II'de listelenmiştir. 6 takım ve 35 familyaya ait 118 tür "Fauna Europaea" veritabanına eklenmiştir. Yedi takımdan 90 türün bilimsel isimleri Fauna Europaea veritabanına göre güncellenmiştir.

Anahtar sözcükler: Insecta, Kopački Rit, Hırvatistan, Avrupa

\footnotetext{
${ }^{1} \mathrm{~J}$ J Strossmayer University of Osijek, Department of Biology, Osijek, Croatia Sorumlu yazar (Corresponding author) e-mail: stjepan@biologija.unios.hr Alınış (Received):01.04.2014 Kabul ediliş (Accepted): 03.07.2014
} 


\section{Introduction}

Insects (Insecta) are the most numerous group of animals in the world, with over one million species that have been described (Price, 1997). Aquatic insects are an important trophic level in wetland systems, since they provide food for several wildlife species, such as fish and waterfowl (Maltchik et al., 2012). Wetlands are habitats with high biodiversity (Mitsch \& Gosselink, 2000). There are five wetlands in Croatia which are on the List of Wetlands of International Importance; and one of them is Kopački rit Nature Park (http://www.dzzp.hr; http://www.ramsar.org). The first systematic studies of the entomofauna of Kopački rit NP began during 1943 as a project of the biological station Albertina, which was a part of the Hungarian National Museum from Budapest, with its center in the castle of Eugene of Savoy in Bilje (Szént-Ivány, 1944). The fauna of Lepidoptera, Hymenoptera, Odonata, and Orthoptera was researched at that time (Szént-Ivány, 1944). These data are cited in many papers and ecological studies which were published even fifty to seventy years afterwards (Mihaljević et al., 1999; Horvatić, 2002). Names of many species in these papers are old, having become synonymous, and are not updated to the current names of species according to the Fauna Europaea database. In many recent publications the old names of species are still used, which causes much confusion with regard to the insect fauna in Croatian wetlands. The aim of this overview is to: i) collect all of the old and recent data to show the richness of entomofauna of the Kopački Rit NP, ii) correct old species names (synonyms) from literature data according to the Fauna Europaea database, and finally iii) use new data as an addition to the Fauna Europaea database.

\section{Material and Methods}

The list of species is based on the literary records and unpublished data. Most of the data were found in various entomological journals such as Albertina, Acta Entomologica Jugoslavica, Acta Entomologica Serbica, Aquatic Insects, Entomologia Croatica, Entomologia Generalis, Journal of Vector Ecology, Natura Croatica, Periodicum biologorum, Poljoprivreda, and Vlinders, master's degree theses (Draganić, 2000; Pulitika, 2000; Turić, 2007; Domić, 2009; Vrućina, 2010; Kovačević, 2011; Kulundžić, 2011; Bistrović, 2012; Kurbalija 2012), PhD thesis (Čerba, 2010), ecological studies (Horvatić, 2002) and books (Mihaljević et al., 1999; Springer et al., 2003). The sources of data for each recorded species are listed in Tables 2-10. The collected data were systematized according to the Fauna Europaea database (http://www.faunaeur.org). The new data for the Fauna Europaea database pertaining to Croatia in tables from 2 to 10 are marked with asterisk $\left({ }^{*}\right)$, while old names (synonyms) are marked with black circle. Some specimens from MSc and PhD thesis are deposited in the insect collections of the Department of Biology in Osijek, whereas other specimens are kept in private collections of MSc and PhD students. The accuracy of identification of the data in master's degree thesis and PhD thesis for many species of insects collected in the area of Kopački rit NP were reviewed by domestic or foreign entomologists. Species from the families Carabidae and Scarabaeidae (Coleoptera) were reviewed by Dr. Lucija Šerić-Jelaska (Faculty of Science University of Zagreb), species from the family Chironomidae were reviewed by Dr. Zlatko Mihaljević (Faculty of Science University of Zagreb), a large number of moths (Lepidoptera, Heterocera) were reviewed by Dr. Iva Mihoci and Dr. Martina Šašić (Croatian Natural History Museum in Zagreb). Species from the suborder Symphyta (Hymenoptera) were reviewed by Mr. Franjo Perović (Croatian Natural History Museum in Zagreb). Louse flies (Hippoboscidae) were reviewed by Dr. T. Trilar (Slovenian Museum of Natural History in Ljubljana), hoverflies (Syrphidae) were reviewed by Dr. Ante Vujić (Faculty of Science University of Novi Sad, Serbia). Other groups of insects from the studied area were identified by the entomologists of the Department of Biology JJ Strossmayer University of Osijek. 
The study area: Kopački Rit Nature Park $\left(45^{\circ} 35^{\prime} \mathrm{N}, 18^{\circ} 51^{\prime} \mathrm{E}\right)$ is one of the best preserved wetlands in Europe (Schneider-Jacoby, 1994). It is located in North-Eastern Croatia, in Baranja, in a corner made by the rivers Drava and Danube. It is oval in shape, $8-10 \mathrm{~km}$ long in the west - east direction and about 7$9 \mathrm{~km}$ in the north - south direction, and its surface area is about $100 \mathrm{~km}^{2}$ (Bognar, 1990). The basic ecological features of this area are provided by the dynamics and intensity of flooding of the rivers Drava and Danube. Depending on the quantity of flood water, sections of the park vary in size, shape, and function. Due to the specific micro-relief and flooding, diverse vegetation developed in the area of Kopački Rit. Those are swamp communities, sedges, and high reed communities on the one side, and flood forests on the other. Different vegetation of Kopački Rit provides favorable conditions for the life of numerous animal groups (Springer et al., 2003).

\section{Results and Discussion}

In the Kopački Rit NP 895 species of insects in 8 orders were identified. The largest number of species, $35.53 \%$ belongs to the order Lepidoptera, followed by Coleoptera with $29.38 \%$, Diptera with $12.40 \%$, and Hymenoptera with $8.82 \%$ (Table 1). Species from these four orders make up $86.13 \%$ of the entomofauna of the Kopački Rit NP. Two species of the total number of recorded insects from the order Coleoptera are classified into higher category of threat according to IUCN Red List categories (IUCN, 1994). The butterflies Lycaena dispar (Haworth) and Phengaris arion (L.) are listed as Near Threatened (NT) (IUCN, 2014) (Table 6). Furthermore, Ph. arion, Colias myrmidone (Esper) and Euphydryas maturna (L.) are listed in the Annexes II and/or IV of the EU Habitats Directive (Van Swaay et al., 2010). However, according to European Red List of Butterflies Ph. arion and C. myrmidone are listed as Endangered (EN) on the European level, while E. maturna is listed as Vulnerable (VU) (Van Swaay et al., 2010). All other butterflies (Table, 6) belong to Least Concern (LC) category (Van Swaay et al., 2010). 43 dragonfly species (Table, 8) belong to the Least Concern (LC) category according to IUCN 2014. Gomphus flavipes (Charpentier), Ophiogomphus cecilia (Fourcroy), Leucorrhinia pectoralis (Charpentier), and Coenagrion ornatum (Selys) are listed in Annex II of the Bern Convention and in the Annexes II and/or IV of the EU Habitats Directive (Kalkman et al., 2010). Six species of European Concern from the order Coleoptera were found in Kopački Rit NP. Graphoderus bilineatus (De Geer) is listed on the IUCN Red List as Vulnerable (VU) and on Annex II of the Bern Convention and Annexes II and IV of the EU Habitats Directive (http://www.iucnredlist.org). Also, according to IUCN (2014) Cerambyx cerdo L. is listed as Vulnerable species (VU) in the World. Elater ferrugineus L., and Lucanus cervus (L.) (Table, 2) are listed as Near Threatened (NT) in the European Red List of Saproxylic Beetles, while Melanotus villosus (Fourcroy) and Dorcus parallelipipedus (L.) are of Least concern (LC) at the European level (Nieto \& Alexander, 2010). On the Fauna Europaea database for Croatia, 118 species of insects classified in 35 families and 6 orders have not been mentioned. The most numerous is the order Lepidoptera with 39 species classified in 11 families, while other orders are represented as follows: Coleoptera with 34 species and 10 families, Diptera with 26 species and 4 families, Hymenoptera with 17 species and 8 families, and the orders Hemiptera and Orthoptera with 1 species and 1 family each (Table, 2-10).

Table 1. List of identified insects in Kopački rit NP

\begin{tabular}{llll}
\hline Order & Suborder & Family & Species \\
\hline Lepidoptera & 0 & 26 & 318 \\
Coleoptera & 2 & 19 & 263 \\
Diptera & 2 & 6 & 111 \\
Hymenoptera & 2 & 10 & 79 \\
Odonata & 2 & 8 & 48 \\
Psocoptera & 2 & 7 & 29 \\
Hemiptera & 1 & 9 & 24 \\
Orthoptera & 2 & 5 & 23 \\
Total: 8 & 13 & 90 & 895 \\
\hline
\end{tabular}


Table 2. List of Coleoptera fauna in Kopački Rit NP

\begin{tabular}{|c|c|c|c|}
\hline Suborder & Family & Species & Source of data \\
\hline \multirow[t]{65}{*}{ Adephaga } & Carabidae & Abax carinatus (Duftschmid 1812) & Tallósi, 2008 \\
\hline & & Abax paralellus (Duftschmid 1812) & Horvatić, 2002 \\
\hline & & Abax parallelepipedus (Piller \& Mitterpacher 1783) & \\
\hline & & Acupalpus parvulus (Sturm 1825) & Tallósi, 2008 \\
\hline & & - Acupalpus dorsalis Fabricius 1787 & \\
\hline & & Agonum hypocrita (Apfelbeck 1904) & \\
\hline & & Agonum longicorne Chaudoir 1846 & \\
\hline & & Agonum lugens (Duftschmid 1812) & Horvatić, 2002 \\
\hline & & Agonum permoestum Puel 1938 & Tallósi, 2008 \\
\hline & & Agonum thoreyi Dejean 1828 & \\
\hline & & Agonum versutum Sturm 1824 & Horvatić, 2002 \\
\hline & & Amara aenea (De Geer 1774) & Mihaljević et al., 1999 \\
\hline & & Amara bifrons (Gyllenhal 1810) & Domić, 2009 \\
\hline & & Amara communis (Panzer 1797) & \\
\hline & & Amara fusca Dejean 1828 & Horvatić, 2002 \\
\hline & & - Amara complanata Dejean 1828 & \\
\hline & & Amara cursitans Zimmerman 1832 & \\
\hline & & - Amara properans Zimerman 1832 & \\
\hline & & Amara ingenua (Duftschmid 1812) & \\
\hline & & Amara nitida Sturm 1825 & Domić, 2009 \\
\hline & & Amara ovata (Fabricius 1792) & \\
\hline & & Amara praetermissa (C.R. Sahlberg 1827) & Horvatić, 2002 \\
\hline & & Amara saphyrea Dejean 1828 & Domić, 2009 \\
\hline & & Amara similata (Gyllenhal 1810) & \\
\hline & & Anchomenus dorsalis (Pontoppidan 1763) & \\
\hline & & • Agonum dorsalis Pontoppidan 1763 & \\
\hline & & Anisodactylus binotatus (Fabricius 1787) & Tallósi, 2008 \\
\hline & & Anisodactylus signatus (Panzer 1796) & Domić, 2009 \\
\hline & & Asaphidion flavipes (Linne 1761) & Mihaljević et al., 1999 \\
\hline & & ${ }^{*}$ Atranus ruficollis (Gautier des Cottes 1857) & Domić, 2009 \\
\hline & & Badister dilatatus Chaudoir 1837 & \\
\hline & & ${ }^{\star}$ Badister unipustulatus Bonelli 1813 & \\
\hline & & Bembidion quadrimaculatum (Linne 1761) & Tallósi, 2008 \\
\hline & & $\begin{array}{l}\text { Bembidion quadripustulatum Audinet-Serville } 1821 \\
{ }^{*} \text { Blemus discus (Fabricius, 1792) }\end{array}$ & \\
\hline & & Brachinus crepitans (Linne 1758) & Domić, 2009 \\
\hline & & Calathus fuscipes (Goeze 1777) & Tallósi, 2008 \\
\hline & & Calathus melanocephalus (Linne 1758) & \\
\hline & & Calosoma inquisitor (Linne 1758) & Domić, 2009 \\
\hline & & Carabus cancellatus Illiger 1798 & \\
\hline & & Carabus clatratus Linne 1761 & \\
\hline & & Carabus coriaceus Linne 1758 & \\
\hline & & Carabus germarii Sturm 1815 & Tallósi, 2008 \\
\hline & & Carabus granulatus Linne 1758 & Domić, 2009 \\
\hline & & Carabus violaceus Linne 1758 & \\
\hline & & Chlaeniellus tristis (Schaller 1783) & Mihaljević et al., 1999 \\
\hline & & Chlaenius spoliatus (P. Rossi 1792) & Tallósi, 2008 \\
\hline & & Cicindela campestris Linne 1758 & Domić, 2009 \\
\hline & & Clivina collaris (Herbst 1784) & \\
\hline & & Clivina fossor (Linne 1758) & Tallósi, 2008 \\
\hline & & ${ }^{\star}$ Clivina laevifrons Chaudoir 1842 & Domić, 2009 \\
\hline & & Cryptophonus melancholicus (Dejean 1829) & \\
\hline & & - Harpalus melancholicus (Dejean 1829) & \\
\hline & & Diachromus germanus (Linne 1758) & \\
\hline & & ${ }^{*}$ Dicheirotrichus placidus (Gyllenhal 1827) & Mihaljević et al., 1999 \\
\hline & & Drypta dentata (P. Rossi 1790) & Tallósi, 2008 \\
\hline & & Dyschiriodes aeneus (Dejean 1825) & \\
\hline & & - Dyschirius aeneus (Dejean 1825) & \\
\hline & & Dyschiriodes chalybaeus (Apfelbeck, 1899) & \\
\hline & & - Dyschirius chalybaeus Apfelbeck 1899 & \\
\hline & & Dyschiriodes globosus (Herbst 1783) & Domić, 2009 \\
\hline & & Elaphrus aureus P. Müller 1821 & \\
\hline & & ${ }^{\star}$ Emphanes minimus (Fabricius 1792) & \\
\hline & & - Bembidion minimus (Fabricius 1792) & \\
\hline & & Emphanes tenellus (Erichson 1837) & Tallósi, 2008 \\
\hline & & - Bembidion tenellum Erichson 1837 & \\
\hline
\end{tabular}


Table 2. List of Coleoptera fauna in Kopački Rit NP (Continued)

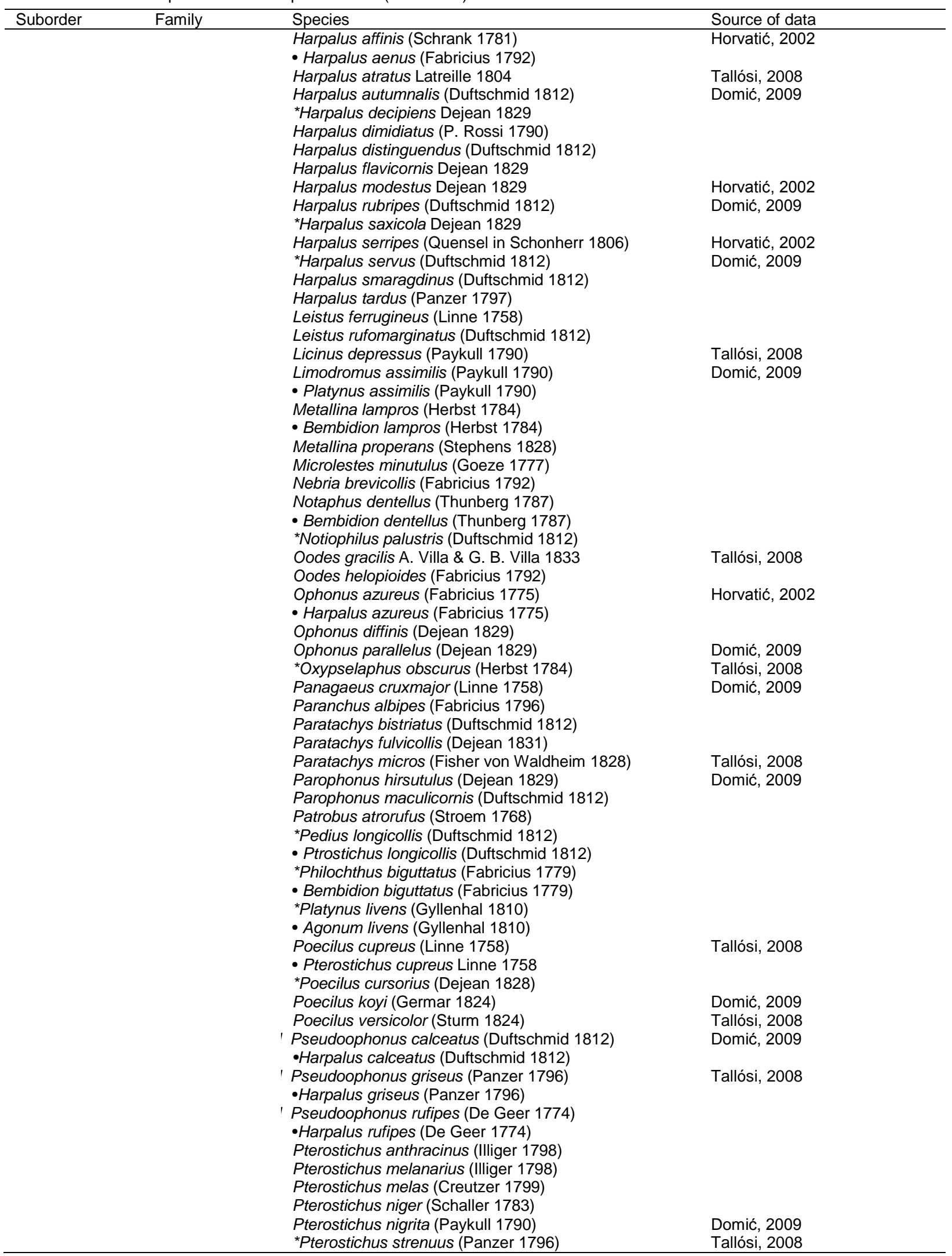


Table 2. List of Coleoptera fauna in Kopački Rit NP (Continued)

\begin{tabular}{|c|c|c|c|}
\hline Suborder & Family & Species & Source of data \\
\hline & \multirow{44}{*}{ Dytiscidae } & Pterostichus vernalis (Panzer 1796) & \\
\hline & & Stenolophus skrimshiranus Stephens 1828 & Domić, 2009 \\
\hline & & $\begin{array}{l}\text { Stenolophus discophorus (Fischer von Waldheim } \\
\text { 1823) }\end{array}$ & Tallósi, 2008 \\
\hline & & Stenolophus mixtus (Herbst 1784) & \multirow{5}{*}{ Domić, 2009} \\
\hline & & Stomis pumicatus (Panzer 1796) & \\
\hline & & Syntomus obscuroguttatus (Duftschmid 1812) & \\
\hline & & Syntomus pallipes (Dejean 1825) & \\
\hline & & Syntomus truncatellus (Linne 1761) & \\
\hline & & Trechus nigrinus Putzeys 1847 & Horvatić, 2002 \\
\hline & & Trechus quadristriatus (Schrank 1781) & Mihaljević et al., 1999; Tallósi, 2008 \\
\hline & & $\begin{array}{l}\text { Trepanes fumigatus (Duftschmid 1812) } \\
\text { - Bembidion fumigatum Duftschmid } 1812\end{array}$ & Tallósi, 2008 \\
\hline & & Trepanes octomaculatus (Goeze 1777) & \multirow[t]{2}{*}{ Domić, 2009} \\
\hline & & - Bembidion octomaculatus (Goeze 1777) & \\
\hline & & Acilius sulcatus (Linnaeus 1758) & Bistrović, 2012 \\
\hline & & Agabus affinis (Paykull 1798) & \\
\hline & & Agabus undulatus (Schrank 1776) & \multirow[t]{2}{*}{ Turić, 2007} \\
\hline & & Colymbetes fuscus (Linnaeus 1758) & \\
\hline & & Copelatus haemorrhoidalis (Fabricius 1787) & Bistrović, 2012 \\
\hline & & Cybister lateralimarginalis (De Geer 1774) & Turić et al., 2012 \\
\hline & & Dytiscus circumcinctus Ahrens 1811 & Bistrović, 2012 \\
\hline & & Dytiscus dimidiatus Bergstrasser 1778 & Mihaljević et al., 1999 \\
\hline & & Dytiscus marginalis Linnaeus 1758 & Turić, 2007 \\
\hline & & Graphoderus austriacus (Sturm 1834) & \\
\hline & & Graphoderus bilineatus (De Geer 1774) & \\
\hline & & Graptodytes bilineatus (Sturm 1835) & \multirow[t]{2}{*}{ Bistrović, 2012} \\
\hline & & Hydaticus seminiger (De Geer 1774) & \\
\hline & & Hydaticus transversalis (Pontoppidan 1763) & \multirow[t]{4}{*}{ Turić, 2007} \\
\hline & & Hydroglyphus geminus (Fabricius 1792) & \\
\hline & & Hydroporus angustatus Sturm 1835 & \\
\hline & & Hydroporus ferrugineus Stephens 1829 & \\
\hline & & Hydroporus palustris (Linnaeus 1761) & Bistrović, 2012 \\
\hline & & Hygrotus impressopunctatus (Schaller 1783) & \multirow{2}{*}{ Turić, 2007} \\
\hline & & Hygrotus inaequalis (Fabricius 1776) & \\
\hline & & Hygrotus parallellogrammus (Ahrens 1812) & Merdić et al., 2005 \\
\hline & & Hygrotus versicolor (Schaller 1783) & Merdić et al., 2005; Turić et al., 2012 \\
\hline & & Hyphydrus anatolicus Guignot 1957 & Turić, 2007 \\
\hline & & Hyphydrus ovatus (Linnaeus 1761) & Bistrović, 2012 \\
\hline & & Ilybius fenestratus (Fabricius 1781) & Turić, 2007; Turić et al., 2012 \\
\hline & & Laccophilus minutus (Linnaeus 1758) & Merdić et al. 2005 \\
\hline & & Laccophilus poecilus Klug 1834 & \multirow[t]{3}{*}{ Turić, 2007} \\
\hline & & Porhydrus lineatus (Fabricius 1775) & \\
\hline & & Rhantus exsoletus (Forster 1771) & \\
\hline & & Rhantus latitans Sharp 1882 & Turić et al., 2012 \\
\hline & & Rhantus suturalis (MacLeay 1825) & Mihaljević et al., 1999 \\
\hline & Haliplidae & Haliplus fluviatilis Aube 1836 & Turić, 2007; Turić et al., 2012 \\
\hline & & *Haliplus furcatus Seidlitz 1887 & Bistrović, 2012 \\
\hline & & Haliplus immaculatus Gerhardt 1877 & Turić, 2007 \\
\hline & & Haliplus ruficollis (De Geer 1774) & Turić et al., 2012 \\
\hline & & Haliplus variegatus Sturm 1834 & Turić, 2007 \\
\hline & & Peltodytes caesus (Duftschmid 1805) & \\
\hline & \multirow[t]{3}{*}{ Noteridae } & Lytta vesicatoria (Linnaeus 1758) & \multirow{13}{*}{$\begin{array}{l}\text { Merdić et al., } 2005 \\
\text { Turić, } 2007 \\
\text { Mihaljević et al., } 1999 \\
\text { Source of data } \\
\text { Mihaljević et al., } 1999\end{array}$} \\
\hline & & Noterus crassicornis (O.F. Müller 1776) & \\
\hline & & Noterus clavicornis (De Geer 1774) & \\
\hline Suborder & Family & Species & \\
\hline \multirow{9}{*}{ Polyphaga } & \multirow[t]{4}{*}{ Aphodiidae } & Acrossus luridus (Fabricius 1775) & \\
\hline & & - Aphodius luridus (Fabricius 1775) & \\
\hline & & Teuchestes fossor (Linnaeus 1758) & \\
\hline & & - Aphodius fossor (Linnaeus 1758) & \\
\hline & \multirow[t]{2}{*}{ Cantharidae } & ${ }^{*}$ Cantharis fusca Linnaeus 1758 & \\
\hline & & *Cantharis livida Linnaeus 1758 & \\
\hline & Cerambycidae & Cerambyx cerdo Linnaeus 1758 & \\
\hline & Coccinellidae & Coccinella septempunctata Linnaeus 1758 & \\
\hline & Curculionidae & *Ceutorhynchus napi Gyllenhal 1837 & \\
\hline
\end{tabular}


Table 2. List of Coleoptera fauna in Kopački Rit NP (Continued)

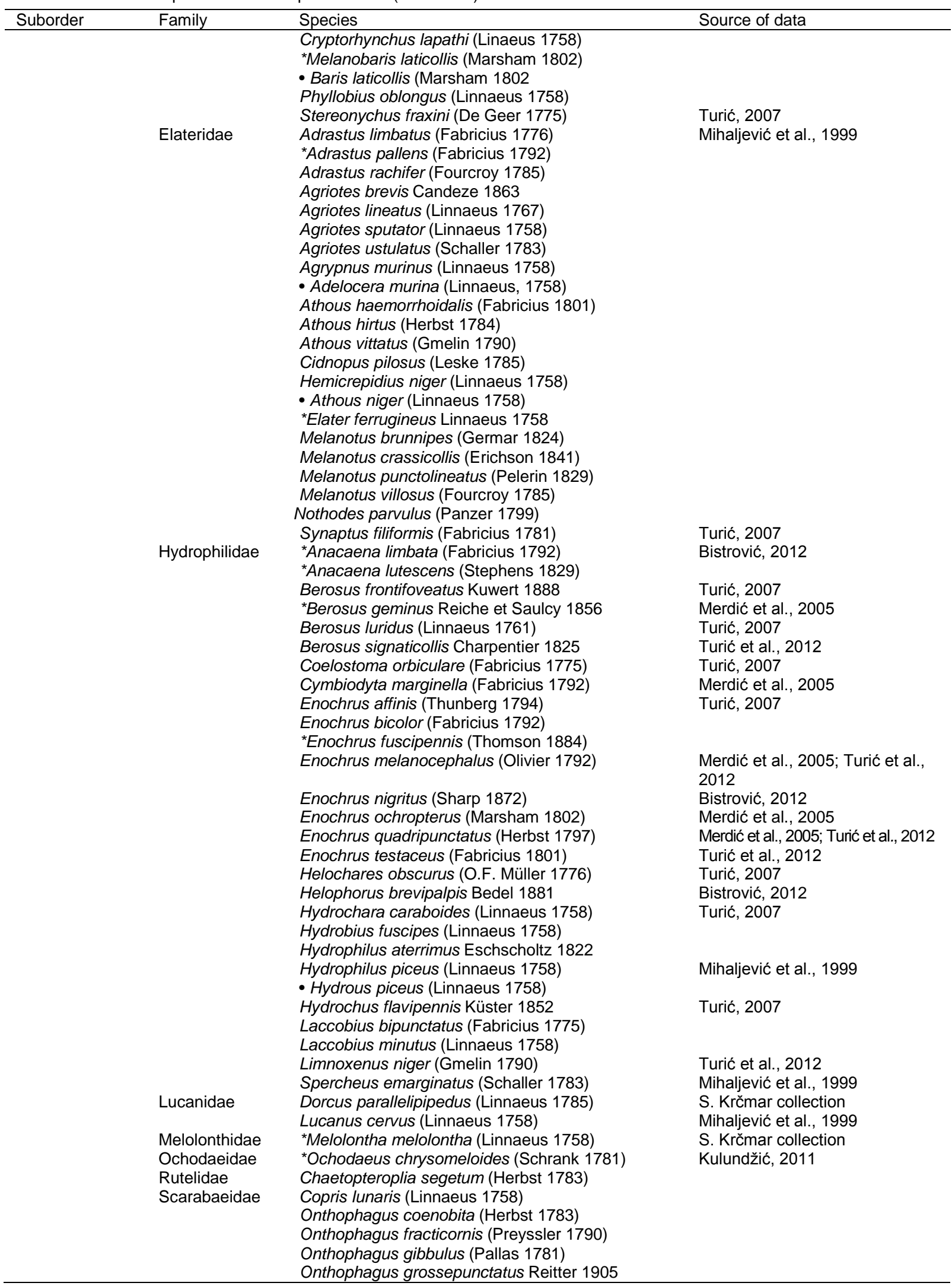


Table 2. List of Coleoptera fauna in Kopački Rit NP (Continued)

\begin{tabular}{|c|c|c|c|}
\hline \multirow[t]{32}{*}{ Suborder } & Family & Species & Source of data \\
\hline & & Onthophagus joannae Goljan 1953 & \\
\hline & & Onthophagus ovatus (Linnaeus 1767) & \\
\hline & & Onthophagus similis (Scriba 1790) & \\
\hline & & Onthophagus vacca (Linnaeus 1767) & \\
\hline & & Onthophagus verticicornis (Laicharting 1781) & \\
\hline & Silphidae & Aclypea undata (O. F. Müller 1776) & Turić, 2007 \\
\hline & & - Blitophaga undata (O. F. Müller 1776) & \\
\hline & Staphylinidae & Gabrius nigritulus (Gravenhorst 1802) & Mihaljević et al., 1999 \\
\hline & & Lathrobium impressum Heer 1841 & \\
\hline & & Ocypus olens (O. Müller 1764) & \\
\hline & & Paederus fuscipes Curtis 1826 & \\
\hline & & Paederus riparius (Linnaeus 1758) & \\
\hline & & Philonthus cognatus Stephens 1832 & \\
\hline & & Philonthus rubripennis (Stephens 1832) & \\
\hline & & Philonthus quisquiliarius (Gyllenhal 1810) & \\
\hline & & Stenus aterrimus Erichson 1839 & \\
\hline & & Stenus comma LeConte 1863 & \\
\hline & & *Stenus crassus Stephens 1833 & \\
\hline & & Stenus humilis Erichson 1839 & \\
\hline & & Stenus palustris Erichson 1839 & \\
\hline & & Tachyporus hypnorum (Fabricius 1775) & \\
\hline & & *Tasgius ater (Gravenhorst 1802) & \\
\hline & & Tetartopeus terminatus (Gravenhorst 1802) & \\
\hline & & - Lathrobium terminatus (Gravenhorst 1802) & \\
\hline & & Xantholinus linearis (Oliver 1795) & \\
\hline & Tenebrionidae & Blaps lethifera Marsham 1802 & \\
\hline & & Blaps mortisaga (Linnaeus 1758) & \\
\hline & & *Hymenalia rufipes (Fabricius 1792) & \\
\hline & & *Omophlus proteus Kirsch 1869 & \\
\hline & & Opatrum sabulosum (Linnaeus 1761) & \\
\hline & & ${ }^{*}$ Pedinus femoralis (Linnaeus 1767) & \\
\hline
\end{tabular}

Legend: * appendix for the Fauna Europaea database, $\bullet$ old names (synonyms)

Table 3. List of Diptera fauna in Kopački Rit NP

\begin{tabular}{|c|c|c|c|}
\hline Suborder & Family & Species & Source of data \\
\hline \multirow[t]{27}{*}{ Brachycera } & Hippoboscidae & $\begin{array}{l}\text { *Hippobosca equina Linnaeus } 1758 \\
\text { *Lipoptena cervi Linnaeus } 1758\end{array}$ & Trilar \& Krčmar, 2005 \\
\hline & Syrphidae & ${ }^{*}$ Callicera spinolae Rondani 1844 & Jeličić et al., 2010 \\
\hline & & Chrysotoxum bicinctum (Linnaeus 1758) & \\
\hline & & Chrysotoxum elegans Loew 1841 & \\
\hline & & Chrysotoxum vernale Loew 1841 & \\
\hline & & ${ }^{*}$ Chrysotoxum verralli Collin 1940 & \\
\hline & & Epistrophe eligans (Harris 1780) & \\
\hline & & Epistrophe melanostoma (Zetterstedt 1843) & \\
\hline & & Episyrphus balteatus (de Geer 1776) & \\
\hline & & Eriozona erratica (Linnaeus 1758) & \\
\hline & & Eristalis arbustorum (Linnaeus 1758) & \\
\hline & & Eristalis pertinax (Scopoli 1763) & \\
\hline & & Eristalis tenax (Linnaeus 1758) & \\
\hline & & Eupeodes corollae (Fabricius 1794) & \\
\hline & & ^Eupeodes lapponicus (Zetterstedt 1838) & \\
\hline & & Eupeodes luniger (Meigen 1822) & \\
\hline & & Ferdinandea cuprea (Scopoli 1763) & \\
\hline & & Ferdinandea ruficornis (Fabricius 1775) & \\
\hline & & Helophilus pendulus (Linnaeus 1758) & \\
\hline & & Helophilus trivittatus (Fabricius 1805) & \\
\hline & & Melanostoma mellinum (Linnaeus 1758) & \\
\hline & & Melanostoma scalare (Fabricius 1794) & \\
\hline & & Meliscaeva auricollis (Meigen 1822) & \\
\hline & & Myathropa florea (Linnaeus 1758) & \\
\hline & & *Paragus pecchiolii Rondani 1857 & \\
\hline & & Platycheirus clypeatus (Meigen 1822) & \\
\hline & & Platycheirus fulviventris (Macquart 1829) & \\
\hline
\end{tabular}


Table 3. List of Diptera fauna in Kopački Rit NP (Continued)

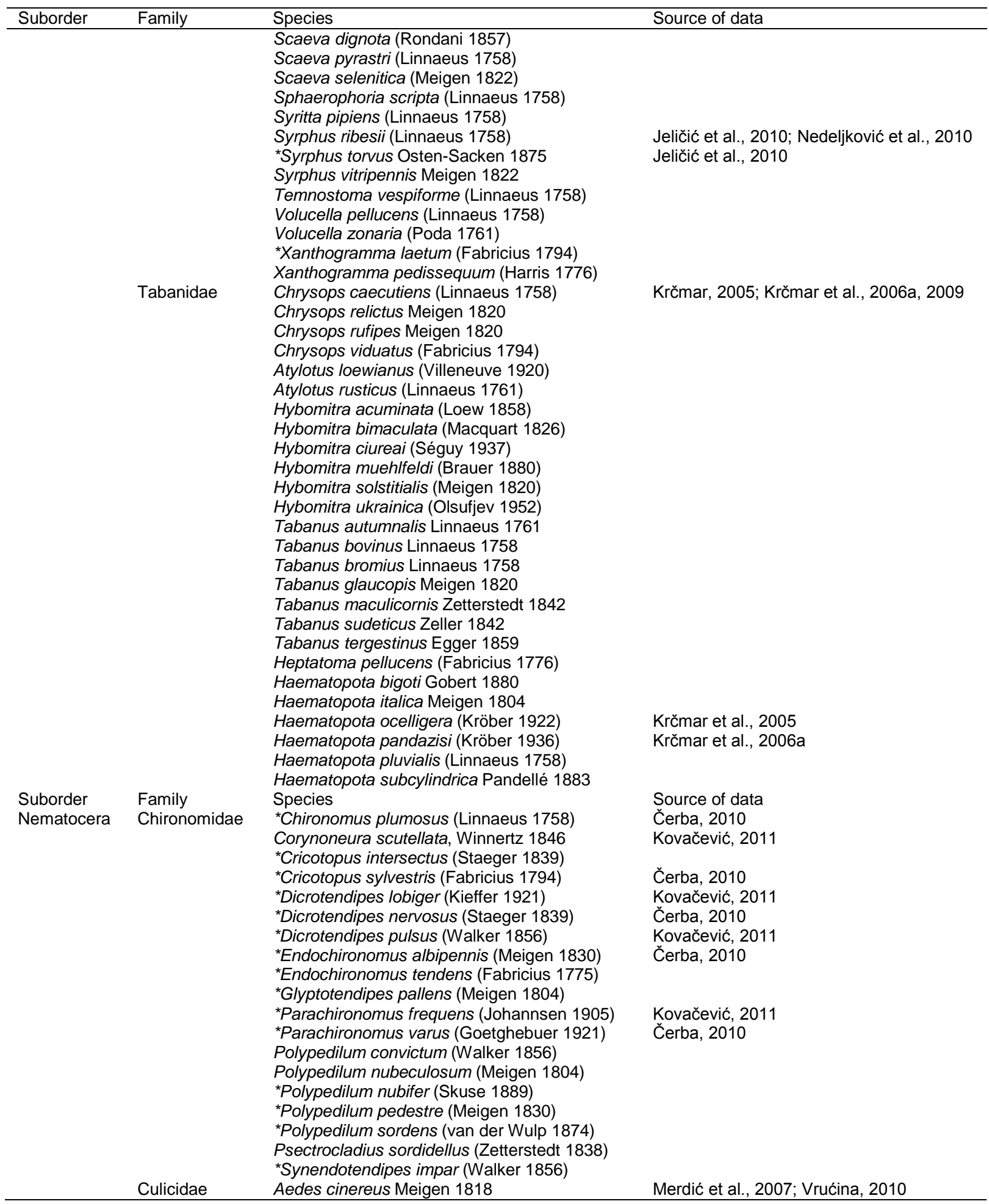


Table 3. List of Diptera fauna in Kopački Rit NP (Continued)

\begin{tabular}{|c|c|c|c|}
\hline \multirow[t]{24}{*}{ Suborder } & Family & Species & Source of data \\
\hline & & Aedes vexans (Meigen 1830) & Merdić, 1993; Merdić \& Sudarić, 2003 \\
\hline & & Anopheles claviger (Meigen 1804) & Merdić \& Sudarić, 2003; Vrućina, 2010 \\
\hline & & Anopheles hyrcanus (Pallas 1771) & \\
\hline & & Anopheles maculipennis Meigen 1818 & Vrućina, 2010 \\
\hline & & Anopheles messeae Falleroni 1926 & $\begin{array}{l}\text { Merdić \& Sudarić, 2003; Merdić et al., } \\
2007\end{array}$ \\
\hline & & Anopheles plumbeus Stephens 1828 & Merdić \& Sudarić, 2003; Vrućina, 2010 \\
\hline & & Coquillettidia richiardii (Ficalbi 1889) & $\begin{array}{l}\text { Merdić \& Sudarić, 2003; Merdić et al., } \\
2007\end{array}$ \\
\hline & & Culex modestus Ficalbi 1890 & \\
\hline & & Culex pipiens Linnaeus 1758 & \\
\hline & & Culex territans Walker 1856 & Vrućina, 2010 \\
\hline & & Culiseta annulata (Schrank 1776) & Merdić \& Sudarić, 2003; Vrućina, 2010 \\
\hline & & Ochlerotatus caspius (Pallas 1771) & Merdić \& Sudarić, 2003; Merdić et al., \\
\hline & & $\begin{array}{l}\text { - Aedes casplus (Pallas 1//1) } \\
\text { Ochlerotatus cantans (Meigen 1818) }\end{array}$ & Merdić et al., 2008 \\
\hline & & Ochlerotatus cataphylla (Dyar 1916) & Vrućina, 2010 \\
\hline & & Ochlerotatus excrucians (Walker 1856) & Merdić \& Sudarić, 2003; Merdić et al., \\
\hline & & - Aedes excrucians (Walker 1856) & 2008 \\
\hline & & Ochlerotatus flavescens (Müller 1764) & Vrućina, 2010 \\
\hline & & $\begin{array}{l}\text { Ochlerotatus geniculatus (Olivier 1791) } \\
\text { - Aedes geniculatus (Olivier 1791) }\end{array}$ & Merdić, 1993 \\
\hline & & Ochlerotatus sticticus (Meigen 1838) & $\begin{array}{l}\text { Merdić \& Sudarić, 2003; Merdić et al., } \\
2007\end{array}$ \\
\hline & & Uranotaenia unguiculata Edwards 1913 & Vrućina, 2010 \\
\hline & Simuliidae & *Simulium balcanicum (Enderlein 1924) & Sudarić - Bogojević et al., 2009 \\
\hline & & *Simulium equinum (Linnaeus 1758) & \\
\hline & & Simulium erythrocephalum (De Geer 1776) & \\
\hline & & *Simulium reptans (Linnaeus 1758) & \\
\hline
\end{tabular}

Legend: * appendix for the Fauna Europaea database, • old names (synonyms)

Table 4. List of Hemiptera fauna in Kopački Rit NP

\begin{tabular}{|c|c|c|c|}
\hline Suborder & Family & Species & Source of data \\
\hline \multirow[t]{24}{*}{ Heteroptera } & Corixidae & Corixa punctata (lliger 1807) & Turić, 2007 \\
\hline & & Cymatia coleoptrata (Fabricius 1777) & \\
\hline & & Hesperocorixa linnaei (Fieber 1848) & \\
\hline & & *Sigara dorsalis (Leach 1817) & \\
\hline & & Sigara falleni (Fieber 1848) & \\
\hline & & Sigara nigrolineata (Fieber 1848) & Turić et al., 2012 \\
\hline & & Sigara semistriata (Fieber 1848) & Turić, 2007 \\
\hline & & Sigara striata (Linnaeus 1758) & \\
\hline & Gerridae & Aquarius najas (De Geer 1773) & \\
\hline & & Aquarius paludum (Fabricius 1794) & Turić et al., 2012 \\
\hline & & Gerris asper (Fieber 1860) & \\
\hline & & Gerris lacustris (Linnaeus 1758) & Turić, 2007 \\
\hline & & Gerris odontogaster (Zetterstedt 1828) & \\
\hline & Hydrometridae & Hydrometra stagnorum (Linnaeus 1758) & \\
\hline & Mesoveliidae & Mesovelia furcata Muslant \& Rey 1852 & \\
\hline & Naucoridae & Ilyocoris cimicoides (Linnaeus 1758) & \\
\hline & Nepidae & $\begin{array}{l}\text { - Naucorls cImicoldes LInnaeus 1/58 } \\
\text { Ranatra linearis (Linnaeus 1758) }\end{array}$ & \\
\hline & & Nepa cinerea Linnaeus 1758 & \\
\hline & Notonectidae & Notonecta glauca Linnaeus 1758 & \\
\hline & & Notonecta maculata Fabricius 1794 & \\
\hline & & Notonecta obliqua Thunberg 1787 & \\
\hline & & Notonecta viridis Delcourt 1909 & Turić et al., 2012 \\
\hline & Pleidae & Plea minutissima Leach 1817 & Turić, 2007 \\
\hline & Veliidae & Microvelia reticulata (Burmeister 1835) & \\
\hline
\end{tabular}

Legend: * appendix for the Fauna Europaea database, • old names (synonyms) 
Table 5. List of Hymenoptera fauna in Kopački Rit NP

\begin{tabular}{|c|c|c|c|}
\hline Suborder & Family & Species & Source of data \\
\hline \multirow[t]{9}{*}{ Apocrita } & Bethylidae & ${ }^{\star}$ Cephalonomia nidicola Szelenyi 1944 & Mihaljević et al., 1999 \\
\hline & Diapriidae & *Trichopria formicaria (Wasmann 1899) & \\
\hline & \multirow[t]{2}{*}{ Eulophidae } & *Aprostocetus longiscapus (Thomson 1878) & \\
\hline & & - Tetrastychus longiscapus (Thomson 1878) & \\
\hline & \multirow[t]{3}{*}{ Pteromalidae } & ${ }^{*}$ Conomorium amplum (Walker 1835) & \\
\hline & & - Conomorium eremita Forster 1841 & \\
\hline & & *Spalangia subpunctata Forster 1850 & \\
\hline & Scelionidae & *Telenomus truncatus (Nees 1834) & \\
\hline & Vespidae & *Vespa crabro Linnaeus 1758 & S. Krčmar collection \\
\hline Suborder & Family & Species & Source of data \\
\hline \multirow[t]{55}{*}{ Symphyta } & \multirow[t]{5}{*}{ Argidae } & Arge cyanocrocea (Förster 1771) & Perović et al., 2006 \\
\hline & & Arge enodis (Linnaeus 1767) & \\
\hline & & Arge melanochra (Gmelin 1790) & \\
\hline & & Arge ochropus (Gmelin 1790) & \\
\hline & & Sterictiphora furcata (Villers 1789) & \\
\hline & \multirow{7}{*}{ Cephidae } & ${ }^{*}$ Calameuta filiformis (Eversmann 1847) & \\
\hline & & *Calameuta haemorrhoidalis (Fabricius 1781) & \\
\hline & & ${ }^{*}$ Cephus nigrinus C.G. Thomson 1871 & \\
\hline & & ${ }^{\star}$ Cephus pygmeus (Linnaeus 1767) & \\
\hline & & ${ }^{*}$ Cephus spinipes (Panzer 1800) & \\
\hline & & *Hartigia nigra (Harris 1776) & \\
\hline & & *Trachelus tabidus (Fabricius 1775) & \\
\hline & \multirow[t]{2}{*}{ Pamphiliidae } & Pamphilius alternans (A. Costa 1860) & \\
\hline & & Pamphilius aurantiacus (Giraud 1857) & \\
\hline & \multirow[t]{41}{*}{ Tenthredinidae } & Aglaostigma aucupariae (Klug 1817) & \\
\hline & & Aglaostigma fulvipes (Scopoli 1763) & \\
\hline & & Allantus melanarius(Klug 1818) & \\
\hline & & Allantus togatus (Panzer 1801) & \\
\hline & & Ametastegia equiseti (Fallén 1808) & \\
\hline & & Ametastegia glabrata (Fallén 1808) & \\
\hline & & *Ametastegia pallipes (Spinola 1808) & \\
\hline & & Athalia ancilla Serville 1823 & \\
\hline & & - Athalia glabricolis C.G. Thompson 1870 & \\
\hline & & Athalia bicolor Serville 1823 & \\
\hline & & Athalia circularis (Klug 1815) & \\
\hline & & Athalia cordata Serville 1823 & \\
\hline & & Athalia rosae (Linnaeus 1758) & \\
\hline & & Athalia rufoscutellata Mocsary 1879 & \\
\hline & & Birka cinereipes (Klug 1816) & \\
\hline & & Cladius pectinicornis (Geoffroy 1785) & \\
\hline & & - Cladius diffornis Panzer 1799 & \\
\hline & & Cladius ulmi (Linnaeus 1758) & \\
\hline & & - Trichiocampus ulmi (Linnaeus 1758) & \\
\hline & & Claremontia brevicornis (Brischke 1883) & \\
\hline & & Dolerus eversmanni W.F.Kirby 1882 & \\
\hline & & *Dolerus gibbosus Hartig 1837 & \\
\hline & & Dolerus haematodes (Schrank 1781) & \\
\hline & & Dolerus vestigialis (Klug 1818) & \\
\hline & & Dulophanes morio (Fabricius 1781) & \\
\hline & & Empria tridens (Konow 1896) & \\
\hline & & Eutomostethus gagathinus (Klug 1816) & \\
\hline & & Macrophya albicincta (Schrank 1776) & \\
\hline & & Macrophya annulata (Geffroy 1785) & \\
\hline & & Macrophya blanda (Fabricius 1775) & \\
\hline & & Macrophya diversipes (Schrank 1782) & \\
\hline & & Macrophya duodecimpunctata (Linnaeus 1758) & \\
\hline & & Macrophya militaris (Klug 1817) & \\
\hline & & Macrophya montana (Scopoli 1763) & \\
\hline & & Macrophya postica (Brulle 1832) & \\
\hline & & Macrophya ribis (Schrank 1781) & \\
\hline & & Metallus pumilus (Klug 1816) & \\
\hline & & Monophadnus spinolae (Klug 1816) & \\
\hline & & Monophadnoides ruficruris (Brulle 1832) & \\
\hline & & Nematus bergmanni Dahlbom 1835 & \\
\hline & & Nematus hypoxanthus Förster 1854 & \\
\hline
\end{tabular}


Table 5. List of Hymenoptera fauna in Kopački Rit NP (Continued)

\begin{tabular}{|c|c|c|c|}
\hline \multirow[t]{21}{*}{ Suborder } & Family & Species & Source of data \\
\hline & & Nematus myosotidis (Fabricius 1804) & \\
\hline & & Nematus wahlbergi C.G.Thomson 1871 & \\
\hline & & Pachyprotasis rapae (Linnaeus 1767) & \\
\hline & & Phymatocera aterrima (Klug 1816) & \\
\hline & & *Pontania proxima (Serville 1823) & \\
\hline & & Pontania viminalis (Linnaeus 1758) & \\
\hline & & Pristiphora aphantoneura (Förster 1854) & \\
\hline & & Pristiphora ruficornis (Olivier 1811) & \\
\hline & & Rhogogaster viridis (Linnaeus 1758) & \\
\hline & & Selandria serva (Fabricius 1793) & \\
\hline & & Taxonus agrorum (Fallén 1808) & \\
\hline & & Tenthredo atra Linnaeus 1758 & \\
\hline & & Tenthredo bifasciata O.F. Muller 1766 & \\
\hline & & Tenthredo livida Linnaeus 1758 & \\
\hline & & Tenthredo scrophulariae Linnaeus 1758 & \\
\hline & & Tenthredo temula Scopoli 1763 & \\
\hline & & Tenthredopsis friesei (Konow 1884) & \\
\hline & & Tenthredopsis litterata (Geoffroy 1785) & \\
\hline & & Tenthredopsis nassata (Linnaeus 1767) & \\
\hline & & Tenthredopsis sordida (Klug 1817) & \\
\hline
\end{tabular}

Legend: * appendix for the Fauna Europaea database, • old names (synonyms)

Summarized data of insect fauna of Kopački Rit NP is hard to compare with other wet habitats in Croatia, mainly due to sporadic entomological studies. However, during the last decades the fauna of carabid beetles (Coleoptera: Carabidae) in areas along the Drava River was studied, and 173 species were recorded (Tallósi, 2008). 38 species of these 173 species were also found in the area of Kopački rit NP. In 2007 along the right side of the Drava River in Croatia within the project "Slovenia-HungaryCroatia Neighbourhood Programme 2004-2006", 78 species of butterflies (Lepidoptera) were found (Ábrahám, 2008). 47 species thereof were also recorded in the butterfly fauna of Kopački rit NP. Among the determined butterflies on the area of Kopački rit NP, C. myrmidone deserves special attention because the last record of this species in Croatia dates from 1989. At that time some specimens were collected in the broaded area of Kopački rit NP (Krčmar et al., 1996). Recently, the Tabanidae fauna of some flooded areas in the middle course of the Drava River, on both the Hungarian and Croatian side was studied, 42 species were determined in this research (Krčmar et al., 2006b), 24 thereof are common in both habitats. Furthermore, the finding of $G$. bilineatus is very important from a nature conservation point of view, because only three old records of this species were known in Croatia before 2005, when the first recent record of $G$. bilineatus was reported from the flooded areas of Kopački rit NP (Turić et al., 2012). Along the Sava River in the area of the Lonjsko Polje Nature Park 98 species from the order Coleoptera were recorded (Durbešić et al., 2000; Brigić et al., 2003; Temunović et al., 2007), 46 of those species appear in the fauna of the Kopački rit NP. Also, 30 species from the Odonata order are common for both areas (Schneider-Jacoby \& Ern, 1993; Hill \& Beinlich, 2000; Bogdanović et al., 2002). During the biodiversity inventory program carried out in the Special Nature Reserve Zasavica in Republic of Serbia, more than 550 species of invertebrates were determined, mainly insects (70\%) (Stanković, 2010). In the territory of Zasavica 72 species and 13 subspecies from the family of ground beetles (Carabidae) were recorded (Čurčić \& Stanković, 2011), 32 species thereof appear in the fauna of Kopački rit NP. Comon species from neighbouring wetlands mostly belong to the following chorotypes: Palaearctic, European and Asiatic-European. During the same research programme Curculionidae were represented by 36 species and Cerambycidae by 30 species in the Coleoptera fauna of Zasavica (Pešić, 2012; Pil \& Stanković, 2006), only 2 species thereof are present in the fauna of Kopački rit NP. Fifty-six species of butterflies were recorded in the Zasavica fauna (Stanković, 2010; Jakšić \& Nahirnić, 2011), from which the most interesting findings are: L. dispar, Satyrium $w$ - album (Knoch), Apatura metis Freyer, Papilio machaon L., Zerynthia polyxena (Denis \& Schiffermüller) and Pieris brassicae (L.) (Stanković, 2011). 
Table 6. List of Lepidoptera (Rhopalocera) fauna in Kopački Rit NP

\begin{tabular}{|c|c|c|}
\hline Family & Species & Source of data \\
\hline \multirow[t]{6}{*}{ Hesperiidae } & $\begin{array}{l}\text { Carcharodus alceae (Esper 1780) } \\
\text { Heteropterus morpheus (Pallas 1771) }\end{array}$ & Reinstra, 2004 \\
\hline & Ochlodes sylvanus (Esper 1778) & Szent-Ivány, 1944; Horvatić, 2002 \\
\hline & Pyrgus malvae (Linnaeus 1758) & Reinstra, 2004 \\
\hline & Spialia orbifer (Hübner 1823) & \\
\hline & Thymelicus sylvestris (Poda 1761) & \\
\hline & - Thymelicus flavus (Brunnich 1763) & \\
\hline \multirow[t]{13}{*}{ Lycaenidae } & Celastrina argiolus (Linnaeus 1758) & \\
\hline & Cupido alcetas (Hoffmannsegg 1804) & Szent-Ivány, 1944; Horvatić, 2002 \\
\hline & Cupido argiades (Pallas 1771) & Reinstra, 2004 \\
\hline & Lycaena dispar (Haworth 1802) & \\
\hline & Lycaena phlaeas (Linnaeus 1761) & \\
\hline & Lycaena tityrus (Poda 1761) & \\
\hline & Favonius quercus (Linnaeus 1758) & \\
\hline & Phengaris arion (Linnaeus 1758) & \\
\hline & Plebejus argus (Linnaeus 1758) & \\
\hline & Plebejus argyrognomon (Bergsträsser 1779) & Szent-Ivány, 1944 \\
\hline & Polyommatus icarus (Rottemburg 1775) & Reinstra, 2004 \\
\hline & Satyrium pruni (Linnaeus 1758) & \\
\hline & Satyrium w-album (Knoch 1782) & \\
\hline \multirow[t]{26}{*}{ Nymphalidae } & Aglais io (Linnaeus 1758) & \\
\hline & - Inachis io Linnaeus 1758 & \\
\hline & Aglais urticae (Linnaeus 1758) & \\
\hline & *Apatura metis Freyer 1829 & Krčmar et al., 1996; Reinstra, 2004 \\
\hline & Aphantopus hyperantus (Linnaeus 1758) & Reinstra, 2004 \\
\hline & Araschnia levana (Linnaeus 1758) & \\
\hline & Argynnis adippe (Denis \& Schiffermüller, 1775) & \\
\hline & Argynnis paphia (Linnaeus 1758) & \\
\hline & Boloria dia (Linnaeus 1767) & \\
\hline & Brenthis daphne (Bergsträsser 1780) & \\
\hline & Brintesia circe (Fabricius 1775) & \\
\hline & Coenonympha glycerion (Borkhausen 1788) & \\
\hline & Coenonympha pamphilus (Linnaeus 1758) & \\
\hline & Euphydryas maturna (Linnaeus 1758) & \\
\hline & Issoria lathonia (Linnaeus 1758) & \\
\hline & Lasiommata maera (Linnaeus 1758) & \\
\hline & Lasiommata megera (Linnaeus 1767) & \\
\hline & Maniola jurtina (Linnaeus 1758) & \\
\hline & Melanargia galathea (Linnaeus 1758) & \\
\hline & Melitaea athalia (Rottemburg 1775) & \\
\hline & Neptis sappho (Pallas 1771) & \\
\hline & Nymphalis xanthomelas (Esper 1781) & \\
\hline & Pararge aegeria (Linnaeus 1758) & \\
\hline & Polygonia c-album (Linnaeus 1758) & \\
\hline & Vanessa atalanta (Linnaeus 1758) & \\
\hline & Vanessa cardui (Linnaeus 1758) & \\
\hline \multirow[t]{3}{*}{ Papilionidae } & Iphiclides podalirius (Linnaeus, 1758) & S. Krčmar collection \\
\hline & Papilio machaon Linnaeus 1758 & Krčmar et al., 1996; Reinstra, 2004 \\
\hline & Zerynthia polyxena (Denis \& Schiffermüller 1775) & Reinstra, 2004 \\
\hline \multirow[t]{12}{*}{ Pieridae } & Anthocharis cardamines (Linnaeus 1758) & \\
\hline & Aporia crataegi (Linnaeus 1758) & \\
\hline & Colias alfacariensis Ribbe 1905 & Horvatić, 2002 \\
\hline & Colias croceus (Fourcroy 1785) & Szent-Ivány, 1944 \\
\hline & Colias hyale (Linnaeus 1758) & \\
\hline & Colias myrmidone (Esper 1781) & Szent-Ivány, 1944; Krčmar et al., 1996 \\
\hline & Gonepteryx rhamni (Linnaeus 1758) & Reinstra, 2004 \\
\hline & Leptidea sinapis (Linnaeus 1758) & Szent-Ivány, 1944; Reinstra, 2004 \\
\hline & Pieris brassicae (Linnaeus 1758) & Reinstra, 2004 \\
\hline & Pieris napi (Linnaeus 1758) & \\
\hline & Pieris rapae (Linnaeus 1758) & Szent-Ivány, 1944 \\
\hline & ${ }^{\star}$ Pontia daplidice (Linnaeus 1758) & \\
\hline Riodinidae & Hamearis lucina (Linnaeus 1758) & Reinstra, 2004 \\
\hline
\end{tabular}


Table 7. List of Lepidoptera (Heterocera) fauna in Kopački Rit NP

\begin{tabular}{|c|c|c|}
\hline Family & Species & Source of data \\
\hline Choreutidae & Anthophila fabriciana (Linnaeus 1767) & Mihaljević et al., 1999 \\
\hline \multirow[t]{3}{*}{ Cossidae } & Cossus cossus(Linnaeus 1758) & Vignjević et al., 2010 \\
\hline & Phragmataecia castaneae (Hübner 1790) & Horvatić, 2002 \\
\hline & Zeuzera pyrina (Linnaeus 1761) & Vignjević et al., 2010 \\
\hline \multirow[t]{24}{*}{ Crambidae } & Agriphila tristella (Denis \& Schiffermüller 1775) & Pulitika, 2000 \\
\hline & Anania hortulata (Linnaeus 1758) & Vignjević et al., 2010 \\
\hline & - Eurrhypara hortulata (Linnaeus 1758) & \\
\hline & $\begin{array}{l}\text { Anania verbascalis (Denis \& Schiffermüller 1775) } \\
\text { Calamotropha paludella (Hübner 1824) }\end{array}$ & \\
\hline & Cataclysta lemnata (Linnaeus 1758) & Mihaljević et al., 1999 \\
\hline & Chilo phragmitella (Hübner 1805) & Vignjević et al., 2010 \\
\hline & Chrysoteuchia culmella (Linnaeus 1758) & Horvatić, 2002 \\
\hline & Crambus perlella (Scopoli 1763) & Mihaljević et al., 1999 \\
\hline & ${ }^{*}$ Crambus uliginosellus Zeller 1850 & \\
\hline & Evergestis extimalis (Scopoli 1763) & Vignjević et al., 2010 \\
\hline & $\begin{array}{l}\text { Evergestis pallidata (Hufnagel 1767) } \\
{ }^{*} \text { Nascia cilialis (Hübner 1796) }\end{array}$ & \\
\hline & Nomophila noctuella (Denis \& Schiffermüller 1775) & Mihaljević et al., 1999 \\
\hline & Nymphula nitidulata (Hufnagel 1767) & Horvatić, 2002 \\
\hline & Ostrinia nubilalis (Hübner 1796) & Vignjević et al., 2010 \\
\hline & - Pyrausta nubilalis (Hübner 1796) & \\
\hline & Parapoynx stratiotata (Linnaeus 1758) & Pulitika, 2000 \\
\hline & Paratalanta hyalinalis (Hübner 1796) & Vignjević et al., 2010 \\
\hline & Pleuroptya ruralis (Scopoli 1763) & \\
\hline & Pyrausta aurata (Scopoli 1763) & \\
\hline & Pyrausta despicata (Scopoli 1763) & Pulitika, 2000 \\
\hline & Pyrausta purpuralis (Linnaeus 1758) & Horvatić, 2002 \\
\hline & Sitochroa palealis (Denis \& Schiffermüller 1775) & Vignjević et al., 2010 \\
\hline & Sitochroa verticalis (Linnaeus 1758) & \\
\hline & Udea ferrugalis (Hübner 1796) & \\
\hline \multirow[t]{6}{*}{ Drepanidae } & Cilix glaucata (Scopoli 1763) & \\
\hline & Habrosyne pyritoides (Hufnagel 1766) & \\
\hline & Tethea ocularis (Linnaeus 1767) & \\
\hline & Thyatira batis (Linnaeus 1758) & \\
\hline & Watsonalla binaria (Hufnagel 1767) & \\
\hline & Watsonalla uncinula (Borkhausen 1790) & \\
\hline \multirow[t]{29}{*}{ Erebidae } & Arctornis I-nigrum (Müller 1764) & \\
\hline & Calliteara pudibunda (Linnaeus 1758) & \\
\hline & Calyptra thalictri (Borkhausen 1790) & \\
\hline & Catocala fraxini (Linnaeus 1758) & Mihaljević et al., 1999 \\
\hline & Catocala nupta (Linnaeus 1767) & Vignjević et al., 2010 \\
\hline & Catocala sponsa (Linnaeus 1767) & \\
\hline & Colobochyla salicalis (Denis \& Schiffermüller 1775) & \\
\hline & Cybosia mesomella (Linnaeus 1758) & Horvatić, 2002 \\
\hline & Diacrisia sannio (Linnaeus 1758) & Vignjević et al., 2010 \\
\hline & Diaphora mendica (Clerck 1759) & \\
\hline & Dysgonia algira (Linnaeus 1767) & Horvatić, 2002 \\
\hline & *Eilema griseola (Hübner 1803) & \\
\hline & Eilema lurideola (Zincken 1817) & Vignjević et al., 2010 \\
\hline & Eilema sororcula (Hufnagel 1766) & Horvatić, 2002 \\
\hline & Euproctis similis (Fuessly 1775) & \\
\hline & Herminia tarsicrinalis (Knoch 1782) & \\
\hline & Herminia tarsipennalis (Treitschke 1835) & Vignjević et al., 2010 \\
\hline & - Zanclognatha tarsipennalis (Treitschke 1835) & \\
\hline & Hypena rostralis (Linnaeus 1758) & \\
\hline & Laelia coenosa (Hübner 1808) & Horvatić, 2002 \\
\hline & Laspeyria flexula (Denis \& Schiffermüller 1775) & Vignjević et al., 2010 \\
\hline & Lithosia quadra (Linnaeus 1758) & \\
\hline & Lygephila craccae (Denis \& Schiffermüller 1775) & \\
\hline & Lygephila pastinum (Treitschke 1826) & \\
\hline & Miltochrista miniata (Forster 1771) & \\
\hline & Orgyia antiqua (Linnaeus 1758) & \\
\hline & *Pelosia muscerda (Hufnagel 1766) & Horvatić, 2002 \\
\hline & Phragmatobia fuliginosa (Linnaeus 1758) & \\
\hline & Phytometra viridaria (Clerck 1759) & Vignjević et al., 2010 \\
\hline
\end{tabular}


Table 7. List of Lepidoptera (Heterocera) fauna in Kopački Rit NP (Continued)

\begin{tabular}{|c|c|c|}
\hline Family & Species & Source of data \\
\hline & Rivula sericealis (Scopoli 1763) & Draganić, 2000 \\
\hline & Schrankia costaestrigalis (Stephens 1834) & Mihaljević et al., 1999 \\
\hline & • Hypenodes costaestrigalis (Stephens 1834) & \\
\hline & Spilosoma lubricipeda (Linnaeus 1758) & Horvatić, 2002 \\
\hline & - Spilosoma menthastri Esper 1786 & \\
\hline & Spilosoma lutea (Hufnagel 1766) & \\
\hline \multirow[t]{2}{*}{ Gelechiidae } & *Anacampsis populella (Clerck, 1759) & Mihaljević et al., 1999 \\
\hline & - Tachyptilia populella (Clerck, 1759) & \\
\hline \multirow[t]{57}{*}{ Geometridae } & *Agriopis aurantiaria (Hübner 1799) & Vignjević et al., 2010 \\
\hline & Agriopis leucophaearia (Denis \& Schiffermüller 1775) & \\
\hline & Agriopis marginaria (Fabricius 1776) & \\
\hline & Alsophila aescularia (Denis \& Schiffermüller 1775) & \\
\hline & *Angerona prunaria (Linnaeus 1758) & \\
\hline & *Apeira syringaria (Linnaeus 1758) & \\
\hline & Apocheima hispidaria (Denis \& Schiffermüller 1775) & \\
\hline & Ascotis selenaria (Denis \& Schiffermüller 1775) & \\
\hline & Biston strataria (Hufnagel 1767) & \\
\hline & *Cabera exanthemata (Scopoli 1763) & \\
\hline & Chiasmia clathrata (Linnaeus 1758) & Mihaljević et al., 1999 \\
\hline & - Semiothisa clathrata (Linnaeus 1758) & \\
\hline & Colostygia pectinataria (Knoch 1781) & Horvatić, 2002 \\
\hline & Colotois pennaria (Linnaeus 1761) & Vignjević et al., 2010 \\
\hline & Comibaena bajularia (Denis \& Schiffermüller 1775) & \\
\hline & Cosmorhoe ocellata (Linnaeus 1758) & \\
\hline & Costaconvexa polygrammata (Borkhausen 1794) & \\
\hline & • Cidaria polygrammaria (Borkhausen 1794) & \\
\hline & Crocallis elinguaria (Linnaeus 1758) & \\
\hline & Cyclophora punctaria (Linnaeus 1758) & \\
\hline & Ecliptopera silaceata (Denis \& Schiffermüller 1775) & \\
\hline & ${ }^{\star}$ Ectropis crepuscularia (Denis \& Schiffermüller 1775) & \\
\hline & ${ }^{*}$ Eilicrinia cordiaria (Hübner 1790) & Horvatić, 2002 \\
\hline & Ematurga atomaria (Linnaeus, 1758) & \\
\hline & ${ }^{\star}$ Ennomos alniaria (Linnaeus 1758) & Vignjević et al., 2010 \\
\hline & *Ennomos autumnaria (Werneburg 1859) & \\
\hline & *Ennomos fuscantaria (Haworth 1809) & \\
\hline & ${ }^{\star}$ Epione repandaria (Hufnagel 1767) & Horvatić, 2002 \\
\hline & Epirrhoe alternata (Müller 1764) & \\
\hline & Epirrita christyi (Allen 1906) & Vignjević et al., 2010 \\
\hline & Epirrita dilutata (Denis \& Schiffermüller 1775) & \\
\hline & Erannis defoliaria (Clerck 1759) & \\
\hline & Gandaritis pyraliata (Denis \& Schiffermüller 1775) & \\
\hline & Hemistola chrysoprasaria (Esper 1793) & \\
\hline & - Hemistola biliosata (Villers 1789) & \\
\hline & Hemithea aestivaria (Hübner 1789) & \\
\hline & Horisme corticata (Treitschke 1835) & Horvatić, 2002 \\
\hline & Horisme radicaria (de La Harpe 1855) & Vignjević et al., 2010 \\
\hline & Horisme tersata (Denis \& Schiffermüller 1775) & \\
\hline & Horisme vitalbata (Denis \& Schiffermüller 1775) & \\
\hline & Hypomecis punctinalis (Scopoli 1763) & \\
\hline & *Hypomecis roboraria (Denis \& Schiffermüller 1775) & \\
\hline & Idaea aversata (Linnaeus 1758) & Horvatić, 2002 \\
\hline & - Sterha aversata (Linnaeus 1758) & \\
\hline & *Idaea biselata (Hufnagel 1767) & \\
\hline & Idaea degeneraria (Hübner 1799) & Vignjević et al., 2010 \\
\hline & Idaea dimidiata (Hufnagel 1767) & Mihaljević et al., 1999 \\
\hline & Idaea muricata (Hufnagel 1767) & Horvatić, 2002 \\
\hline & *Idaea straminata (Borkhausen 1794) & Vignjević et al., 2010 \\
\hline & *Isturgia arenacearia (Denis \& Schiffermüller 1775) & Horvatić, 2002 \\
\hline & - Tephrina arenacearia (Denis \& Schiffermüller 1775) & \\
\hline & Ligdia adustata (Denis \& Schiffermüller 1775) & \\
\hline & *Lomaspilis marginata (Linnaeus 1758) & Vignjević et al., 2010 \\
\hline & *Lomaspilis opis Butler 1878 & Horvatić, 2002 \\
\hline & Lycia hirtaria (Clerck 1759) & Vignjević et al., 2010 \\
\hline & Lythria purpuraria (Linnaeus 1758) & Mihaljević et al., 1999 \\
\hline & *Macaria alternata (Denis \& Schiffermüller 1775) & Vignjević et al., 2010 \\
\hline
\end{tabular}


Table 7. List of Lepidoptera (Heterocera) fauna in Kopački Rit NP (Continued)

\begin{tabular}{|c|c|c|}
\hline \multirow[t]{17}{*}{ Family } & Species & Source of data \\
\hline & Operophtera brumata (Linnaeus 1758) & \\
\hline & Peribatodes rhomboidaria (Denis \& Schiffermüller 1775) & \\
\hline & - Boarmia rhomboidaria (Denis \& Schiffermüller 1775) & \\
\hline & Perizoma lugdunaria (Herrich-Schäffer 1855) & \\
\hline & ${ }^{*}$ Phigalia pilosaria (Denis \& Schiffermüller 1775) & \\
\hline & Rhodometra sacraria (Linnaeus 1767) & \\
\hline & Scopula nigropunctata (Hufnagel 1767) & Horvatić, 2002 \\
\hline & Scopula ornata (Scopoli 1763) & Mihaljević et al., 1999 \\
\hline & Scopula rubiginata (Hufnagel 1767) & \\
\hline & *Selenia dentaria (Fabricius 1775) & Vignjević et al., 2010 \\
\hline & Selenia Iunularia (Hübner 1788) & \\
\hline & *Selenia tetralunaria (Hufnagel 1767) & \\
\hline & Siona lineata (Scopoli 1763) & Horvatić, 2002 \\
\hline & Thalera fimbrialis (Scopoli 1763) & Vignjević et al., 2010 \\
\hline & *Theria rupicapraria (Denis \& Schiffermüller 1775) & \\
\hline & Timandra comae Schmidt 1931 & \\
\hline & *Timandra griseata Petersen 1902 & Mihaljević et al., 1999 \\
\hline \multirow{8}{*}{ Lasiocampidae } & Triodia sylvina (Linnaeus 1761) & \\
\hline & - Hepialus sylvinus (Linnaeus 1761) & \\
\hline & Euthrix potatoria (Linnaeus 1758) & \\
\hline & Gastropacha populifolia (Denis \& Schiffermüller 1775) & \\
\hline & Gastropacha quercifolia (Linnaeus 1758) & \\
\hline & Lasiocampa quercus (Linnaeus 1758) & \\
\hline & Odonestis pruni (Linnaeus 1758) & \\
\hline & Poecilocampa populi (Linnaeus 1758) & \\
\hline Limacodidae & Apoda limacodes (Hufnagel 1766) & \\
\hline \multirow[t]{39}{*}{ Noctuidae } & Acontia lucida (Hufnagel 1766) & \\
\hline & - Amathes lucida (Hufnagel 1766) & \\
\hline & Acontia trabealis (Scopoli 1763) & \\
\hline & - Emmelia trabealis (Scopoli 1763) & \\
\hline & Acronicta rumicis (Linnaeus 1758) & Draganić, 2000 \\
\hline & Actinotia polyodon (Clerck 1759) & Vignjević et al., 2010 \\
\hline & Aedia leucomelas (Linnaeus 1758) & Draganić, 2000 \\
\hline & ${ }^{*}$ Agrochola circellaris (Hufnagel 1766) & Vignjević et al., 2010 \\
\hline & Agrochola litura (Linnaeus 1758) & \\
\hline & Agrotis exclamationis (Linnaeus 1758) & Horvatić, 2002 \\
\hline & Agrotis ipsilon (Hufnagel 1766) & Szent-Ivány, 1944 \\
\hline & Agrotis segetum (Denis \& Schiffermüller 1775) & \\
\hline & Allophyes oxyacanthae (Linnaeus 1758) & Vignjević et al., 2010 \\
\hline & Amphipyra berbera Rungs 1949 & \\
\hline & Amphipyra livida (Denis \& Schiffermüller 1775) & \\
\hline & Amphipyra pyramidea (Linnaeus 1758) & Horvatić, 2002 \\
\hline & - Pyramidcampa pyramidea (Linnaeus 1758) & \\
\hline & Amphipyra tragopoginis (Clerck 1759) & Vignjević et al., 2010 \\
\hline & Anarta trifolii (Hufnagel 1766) & Mihaljević et al., 1999 \\
\hline & - Discestra trifolii (Hufnagel 1766) & \\
\hline & Anorthoa munda (Denis \& Schiffermüller 1775) & Vignjević et al., 2010 \\
\hline & - Perigrapha munda (Denis \& Schiffermüller 1775) & \\
\hline & Autographa gamma (Linnaeus 1758) & Horvatić, 2002 \\
\hline & - Phytometra gamma (Linnaeus 1758) & \\
\hline & Axylia putris (Linnaeus 1761) & \\
\hline & Brachionycha nubeculosa (Esper 1785) & Vignjević et al., 2010 \\
\hline & Caradrina kadenii Freyer 1836 & Mihaljević et al., 1999 \\
\hline & - Elaphria kadenii Freyer 1836 & \\
\hline & Caradrina morpheus (Hufnagel 1766) & Horvatić, 2002 \\
\hline & Charanyca trigrammica (Hufnagel 1766) & \\
\hline & Colocasia coryli (Linnaeus 1758) & Vignjević et al., 2010 \\
\hline & Conisania luteago (Denis \& Schiffermüller 1775) & Horvatić, 2002 \\
\hline & • Hadena luteago (Denis \& Schiffermüller 1775) & \\
\hline & Conistra ligula (Esper 1791) & Vignjević et al., 2010 \\
\hline & Conistra rubiginosa (Scopoli 1763) & \\
\hline & Conistra vaccinii (Linnaeus 1761) & Szent-Ivány, 1944; Mihaljević et al., 1999 \\
\hline & Cosmia pyralina (Denis \& Schiffermüller 1775) & Vignjević et al., 2010 \\
\hline & Craniophora ligustri (Denis \& Schiffermüller 1775) & \\
\hline & Deltote pygarga (Hufnagel 1766) & \\
\hline
\end{tabular}


Table 7. List of Lepidoptera (Heterocera) fauna in Kopački Rit NP (Continued)

\begin{tabular}{|c|c|c|}
\hline Family & Species & Source of data \\
\hline & Diachrysia chrysitis (Linnaeus 1758) & Draganić, 2000 \\
\hline & *Diachrysia stenochrysis (Warren 1913) & Vignjević et al., 2010 \\
\hline & Diloba caeruleocephala Linnaeus 1758 & Draganić, 2000 \\
\hline & Elaphria venustula (Hübner 1790) & Vignjević et al., 2010 \\
\hline & Eucarta amethystina (Hübner 1803) & \\
\hline & Eucarta virgo (Treitschke 1835) & Horvatić, 2002 \\
\hline & •Cologonia virgo (Treitschke 1835) & \\
\hline & Eugnorisma depuncta (Linnaeus 1761) & Mihaljević et al., 1999 \\
\hline & •Rhyacia depuncta (Linnaeus 1761) & \\
\hline & Euplexia lucipara (Linnaeus 1758) & Vignjević et al., 2010 \\
\hline & Eupsilia transversa (Hufnagel 1766) & \\
\hline & Gortyna flavago (Denis \& Schiffermüller 1775) & Draganić, 2000 \\
\hline & Heliothis peltigera (Denis \& Schiffermüller 1775) & Horvatić, 2002 \\
\hline & •Chloridea peltigera (Denis \& Schiffermüller 1775) & \\
\hline & Hoplodrina ambigua (Denis \& Schiffermüller 1775) & \\
\hline & Hoplodrina blanda (Denis \& Schiffermüller 1775) & \\
\hline & Lacanobia oleracea (Linnaeus 1758) & Vignjević et al., 2010 \\
\hline & Lacanobia w-latinum (Hufnagel 1766) & \\
\hline & Leucania obsoleta (Hübner 1803) & Horvatić, 2002 \\
\hline & Lithophane ornitopus (Hufnagel 1766) & Vignjević et al., 2010 \\
\hline & *Longalatedes elymi (Treitschke 1825) & \\
\hline & - Chortodes elymi (Treitschke 1825) & \\
\hline & Macdunnoughia confusa (Stephens 1850) & Horvatić, 2002 \\
\hline & Mamestra brassicae (Linnaeus 1758) & Vignjević et al., 2010 \\
\hline & Moma alpium (Osbeck 1778) & \\
\hline & Mythimna albipuncta (Denis \& Schiffermüller 1775) & Horvatić, 2002 \\
\hline & Mythimna conigera (Denis \& Schiffermüller 1775) & \\
\hline & Mythimna ferrago (Fabricius 1787) & Vignjević et al., 2010 \\
\hline & Mythimna L-album (Linnaeus 1767) & Szent-Ivány, 1944; Mihaljević et al., 1999 \\
\hline & •Hyphilare L-album (Linnaeus 1767) & \\
\hline & Mythimna pallens (Linnaeus 1758) & \\
\hline & -Sideridis pallens Linnaeus 1758) & \\
\hline & Mythimna turca (Linnaeus 1761) & Horvatić, 2002 \\
\hline & Mythimna vitellina (Hübner 1808) & \\
\hline & Noctua fimbriata (Schreber 1759) & Vignjević et al., 2010 \\
\hline & Noctua janthina Denis \& Schiffermüller 1775 & \\
\hline & Noctua pronuba ( Linnaeus 1758) & Szent-Ivány, 1944 \\
\hline & -Tryphaena pronuba (Linnaeus 1758) & \\
\hline & *Nonagria typhae (Thunberg 1784) & Vignjević et al., 2010 \\
\hline & Ochropleura plecta (Linnaeus 1761) & \\
\hline & Orthosia cerasi (Fabricius 1775) & \\
\hline & Orthosia gothica (Linnaeus 1758) & \\
\hline & Orthosia incerta (Hufnagel 1766) & \\
\hline & Orthosia populeti (Fabricius 1775) & \\
\hline & Peridroma saucia (Hübner 1808) & Vignjević et al., 2010 \\
\hline & Phlogophora meticulosa (Linnaeus 1758) & Draganić, 2000 \\
\hline & •Trigonophora meticulosa (Linnaeus 1758) & \\
\hline & Polia nebulosa (Hufnagel 1766) & Vignjević et al., 2010 \\
\hline & Pseudeustrotia candidula (Denis \& Schiffermüller 1775) & \\
\hline & Sideridis rivularis (Fabricius 1775) & Vignjević et al., 2010 \\
\hline & •Hadena rivularis (Fabricius 1775) & \\
\hline & Thalpophila matura (Hufnagel 1766) & Horvatić, 2002 \\
\hline & Tiliacea aurago (Denis \& Schiffermüller 1775) & Vignjević et al., 2010 \\
\hline & Trachea atriplicis (Linnaeus 1758) & \\
\hline & Tyta luctuosa (Denis \& Schiffermüller 1775) & Horvatić, 2002 \\
\hline & Valeria oleagina (Denis \& Schiffermüller 1775) & Vignjević et al., 2010 \\
\hline & Xanthia icteritia (Hufnagel 1766) & \\
\hline & Xestia c-nigrum (Linnnaeus 1758) & Szent-Ivány, 1944 \\
\hline & •Diarsia c-nigrum (Linnnaeus 1758) & \\
\hline & Xestia xanthographa (Denis \& Schiffermüller 1775) & Vignjević et al., 2010 \\
\hline & •Diarsia xanthographa (Denis \& Schiffermüller 1775) & \\
\hline Nolidae & Earias clorana (Linnaeus 1761) & Horvatić, 2002 \\
\hline & Meganola albula (Denis \& Schiffermüller 1775) & Vignjević et al., 2010 \\
\hline & Pseudoips prasinana (Linnaeus 1758) & Draganić, 2000 \\
\hline
\end{tabular}


Table 7. List of Lepidoptera (Heterocera) fauna in Kopački Rit NP (Continued)

\begin{tabular}{|c|c|c|}
\hline Family & Species & Source of data \\
\hline \multirow[t]{11}{*}{ Notodontidae } & Clostera anastomosis (Linnaeus 1758) & Vignjević et al., 2010 \\
\hline & Clostera curtula (Linnaeus 1758) & Horvatić, 2002 \\
\hline & Clostera pigra (Hufnagel 1766) & \\
\hline & Furcula furcula (Clerck 1759) & \\
\hline & Notodonta ziczac (Linnaeus 1758) & Vignjević et al., 2010 \\
\hline & Pheosia tremula (Clerck, 1759) & \\
\hline & Pterostoma palpina (Clerck, 1759) & \\
\hline & Ptilodon capucina (Linnaeus 1758) & \\
\hline & Ptilodon cucullina (Denis \& Schiffermüller 1775) & \\
\hline & ${ }^{*}$ Ptilophora plumigera (Denis \& Schiffermüller 1775) & \\
\hline & Stauropus fagi (Linnaeus 1758) & \\
\hline Plutellidae & Plutella xylostella (Linnaeus 1758) & Mihaljević et al., 1999 \\
\hline \multirow[t]{2}{*}{ Pterophoridae } & Emmelina monodactyla (Linnaeus 1758) & Vignjević et al., 2010 \\
\hline & Pterophorus pentadactyla (Linnaeus 1758) & \\
\hline \multirow[t]{6}{*}{ Pyralidae } & *Dioryctria simplicella Heinemann 1863 & \\
\hline & Endotricha flammealis (Denis \& Schiffermüller 1775) & \\
\hline & Hypsopygia costalis (Fabricius 1775) & Pulitika, 2000 \\
\hline & Oncocera semirubella (Scopoli 1763) & Horvatić, 2002 \\
\hline & •Salebria semirubella (Scopoli 1763) & \\
\hline & *Sciota adelphella (Fischer v. Röslerstamm 1836) & Vignjević et al., 2010 \\
\hline \multirow[t]{2}{*}{ Saturniidae } & Antheraea yamamai (Guérin-Meneville, 1861) & \\
\hline & Saturnia pyri (Denis \& Schiffermüller 1775) & \\
\hline \multirow[t]{7}{*}{ Sphingidae } & Agrius convolvuli (Linnaeus 1758) & Vignjević et al., 2010 \\
\hline & Deilephila porcellus (Linnaeus 1758) & Horvatić, 2002 \\
\hline & Laothoe populi (Linnaeus 1758) & \\
\hline & Macroglossum stellatarum (Linnaeus 1758) & Szent-Ivány, 1944; Mihaljević et al., 1999 \\
\hline & Mimas tiliae (Linnaeus 1758) & Vignjević et al., 2010 \\
\hline & Smerinthus ocellata (Linnaeus 1758) & Horvatić, 2002 \\
\hline & Sphinx ligustri Linnaeus 1758 & Vignjević et al., 2010 \\
\hline \multirow[t]{2}{*}{ Tortricidae } & ${ }^{*}$ Clepsis rurinana (Linnaeus 1758) & Mihaljević et al., 1999 \\
\hline & *Pandemis cerasana (Hübner 1786) & \\
\hline Yponomeutidae & *Yponomeuta padella (Linnaeus 1758) & \\
\hline
\end{tabular}

All of these butterflies were also identified in the areas of Kopački rit NP. Also in the Zasavica reserve, 37 species from the order Odonata were recorded (Stanković, 2010) thereof sighting of Epitheca bimaculata (Charpentier) and G. flavipes are among the most interesting for both wetlands from neighbouring countries represented on the Ramsar List. The latest comparison of the insect fauna from Kopački Rit with Ramsar areas of neighboring countries was carried out with Obedska Bara Special Nature Reserve in Serbia. 93 species from the family Syrphidae were recorded on the same area (Radenković et al., 2004), 5 of those species appear in the fauna of the Kopački rit NP. Also, 6 species from the Culicidae family are comon for both areas (Adamović, 1975; Merdić, 1993). Some recorded species from the orders Coleoptera, Lepidoptera and Odonata belong to different categories of threat and have high faunal and nature conservation value on international level (Table 2, 6, 8) (Kalkman et al., 2010; Nieto \& Alexander, 2010; Van Swaay et al., 2010). 
Table 8. List of Odonata fauna in Kopački Rit NP

\begin{tabular}{|c|c|c|c|}
\hline Suborder & Family & Species & Source of data \\
\hline \multirow[t]{30}{*}{ Anisoptera } & Aeshnidae & Aeshna affinis Vander Linden 1820 & Bogdanović et al., 2002 \\
\hline & & Aeshna mixta Latreille 1805 & \\
\hline & & Aeshna isosceles (Müller 1767) & \\
\hline & & Anax imperator Leach 1815 & \\
\hline & & Anax parthenope (Selys 1839) & \\
\hline & & Brachytron pratense (Müller 1764) & \\
\hline & & Hemianax ephippiger (Burmeister 1839) & \\
\hline & Corduliidae & Cordulia aenea (Linnaeus 1758) & \\
\hline & & Epitheca bimaculata (Charpentier 1825) & \\
\hline & & Somatochlora flavomaculata (Vander Linden 1825) & \\
\hline & & Somatochlora metallica (Vander Linden 1825) & \\
\hline & Gomphidae & Gomphus flavipes (Charpentier 1825) & \\
\hline & & Gomphus vulgatissimus (Linnaeus 1758) & \\
\hline & & Ophiogomphus cecilia (Fourcroy 1785) & \\
\hline & Libellulidae & Crocothemis erythraea (Brullé 1832) & \\
\hline & & Leucorrhinia pectoralis (Charpentier 1825) & \\
\hline & & Libellula depressa Linnaeus 1758 & \\
\hline & & Libellula fulva Müller 1764 & \\
\hline & & Libellula quadrimaculata Linnaeus 1758 & \\
\hline & & Orthetrum albistylum (Selys 1848) & \\
\hline & & Orthetrum cancellatum (Linnaeus 1758) & \\
\hline & & Orthetrum coerulescens (Fabricius 1798) & \\
\hline & & Sympetrum danae (Sulzer 1776) & \\
\hline & & Sympetrum depressiusculum (Selys, 1841) & \\
\hline & & Sympetrum flaveolum (Linnaeus 1758) & \\
\hline & & Sympetrum fonscolombii (Selys 1840) & \\
\hline & & Sympetrum meridionale (Selys 1841) & \\
\hline & & Sympetrum sanquineum (Müller 1764) & \\
\hline & & Sympetrum striolatum (Charpentier 1840) & \\
\hline & & Sympetrum vulgatum (Linnaeus 1758) & \\
\hline \multirow[t]{18}{*}{ Zygoptera } & Calopterygidae & Calopteryx splendens (Harris 1782) & Bogdanović et al., 2002 \\
\hline & & Calopteryx virgo (Linnaeus 1758) & \\
\hline & Coenagrionidae & Coenagrion ornatum (Selys 1850) & \\
\hline & & Coenagrion puella (Linnaeus 1758) & \\
\hline & & Coenagrion pulchellum (Vander Linden 1825) & \\
\hline & & Enallagma cyathigerum (Charpentier 1840) & \\
\hline & & Erythromma najas (Hansemann 1823) & \\
\hline & & Erythromma viridulum (Charpentier 1840) & \\
\hline & & Ischnura elegans (Vander Linden 1820) & \\
\hline & & Ischnura pumilio (Charpentier 1825) & \\
\hline & Lestidae & Lestes barbarus (Fabricius 1798) & \\
\hline & & Lestes dryas Kirby 1890 & \\
\hline & & Lestes macrostigma (Eversmann 1836) & \\
\hline & & Lestes sponsa (Hansemann 1823) & \\
\hline & & Lestes virens (Charpentier 1825) & \\
\hline & & Lestes viridis (Vander Linden 1825) & \\
\hline & & Sympecma fusca (Vander Linden 1820) & \\
\hline & Platycnemididae & Platycnemis pennipes (Pallas 1771) & \\
\hline
\end{tabular}


Surprisingly, 118 species are not mentioned in the Fauna Europaea database for Croatia, even though they have been identified and mentioned in several scientific papers and ecological studies. The most probable reason for that is the fact that these papers, as well as ecological studies, were written in the Croatian language and are therefore not accessible to the wider foreign scientific community. The present list of the insect fauna of Kopački Rit NP does not represent the final status. In further studies more species may be expected in the Kopački Rit NP, which are already known in neighboring areas.

Table 9. List of Orthoptera fauna in Kopački Rit NP

\begin{tabular}{|c|c|c|c|}
\hline Suborder & Family & Species & Source of data \\
\hline \multirow[t]{26}{*}{ Caelifera } & \multirow[t]{24}{*}{ Acrididae } & Aiolopus thalassinus (Fabricius 1781) & \multirow[t]{24}{*}{ Mihaljević et al., 1999} \\
\hline & & - Epacromia thalassina (Fabricius 1781) & \\
\hline & & Calliptamus italicus (Linnaeus 1758) & \\
\hline & & - Caloptenus italicus (Linnaeus 1758) & \\
\hline & & Chorthippus albomarginatus (De Geer 1773) & \\
\hline & & - Chorthippus elegans (Charpentier 1825) & \\
\hline & & Chorthippus biguttulus (Linnaeus 1758) & \\
\hline & & - Stauroderus biguttulus (Linnaeus 1758) & \\
\hline & & Chorthippus dorsatus (Zetterstedt 1821) & \\
\hline & & Chorthippus parallelus (Zetterstedt 1821) & \\
\hline & & - Chorthippus longicornis (Latreille 1804) & \\
\hline & & *Dociostaurus brevicollis (Eversmann 1848) & \\
\hline & & Euthystira brachyptera (Ocskay 1826) & \\
\hline & & - Chrysochraon brachyptera Ocskay 1826 & \\
\hline & & Gomphocerippus rufus (Linnaeus 1758) & \\
\hline & & Mecostethus parapleurus (Hagenbach 1822) & \\
\hline & & Myrmeleotettix maculatus (Thunberg 1815) & \\
\hline & & - Gomphocerus maculatus Thunberg 1815 & \\
\hline & & Omocestus rufipes (Zetterstedt 1821) & \\
\hline & & - Gryllus rufipes (Zetterstedt 1821) & \\
\hline & & Omocestus viridulus (Linnaeus 1758) & \\
\hline & & Pezotettix giornae (Rossi 1794) & \\
\hline & & - Platyphyma giornae (Rossi 1794) & \\
\hline & & Stethophyma grossum (Linnaeus 1758) & \\
\hline & \multirow[t]{2}{*}{ Tetrigidae } & Tetrix bipunctata (Linnaeus 1758) & \\
\hline & & Tetrix subulata (Linnaeus 1758) & \\
\hline Suborder & Family & Species & Source of data \\
\hline \multirow[t]{8}{*}{ Enisfera } & \multirow[t]{2}{*}{ Conocephalidae } & Conocephalus dorsalis (Latreille 1804) & \multirow[t]{8}{*}{ Mihaljević et al., 1999} \\
\hline & & - Xiphidium dorsale Latreille 1804 & \\
\hline & \multirow[t]{2}{*}{ Phaneropteridae } & Phaneroptera falcata (Poda 1761) & \\
\hline & & Poecilimon gracilis (Fieber 1853) & \\
\hline & \multirow[t]{4}{*}{ Tettigoniidae } & Decticus verrucivorus (Linnaeus 1785) & \\
\hline & & Pholidoptera griseoaptera (De Geer 1773) & \\
\hline & & Tettigonia viridissima (Linnaeus 1758) & \\
\hline & & - Locusta viridissima (Linnaeus 1758) & \\
\hline
\end{tabular}


Table 10. List of Psocoptera fauna in Kopački Rit NP

\begin{tabular}{|c|c|c|c|}
\hline Suborder & Family & Species & Source of data \\
\hline \multirow[t]{28}{*}{ Psocomorpha } & Caeciliusidae & Caecilius fuscopterus (Latreille 1799) & $\begin{array}{l}\text { Kalinović et al., 1980; } \\
\text { Mihaljević et al., } 1999\end{array}$ \\
\hline & & Valenzuela atricornis (McLachlan 1869) & \\
\hline & & - Caecilius atricornis McLachlan 1869 & \\
\hline & & Valenzuela corsicus (Kolbe 1882) & \\
\hline & & - Caecilius rhenanus Tetens 1891 & \\
\hline & & Valenzuela flavidus (Stephens 1836) & \\
\hline & & - Caecilius flavidus (Stephens 1836) & \\
\hline & & Valenzuela piceus (Kolbe 1882) & \\
\hline & & - Caecilius piceus Kolbe 1882 & \\
\hline & Ectopsocidae & Ectopsocopsis cryptomeriae (Enderlein 1907) & \\
\hline & & Ectopsocus meridionalis Ribaga 1904 & \\
\hline & Lachesillidae & Lachesilla bernardi Badonnel 1938 & \\
\hline & & Lachesilla pedicularia (Linnaeus 1758) & \\
\hline & & Lachesilla quercus (Kolbe 1880) & \\
\hline & Peripsocidae & Peripsocus alboguttatus (Dalman 1823) & \\
\hline & & Peripsocus parvulus Kolbe 1880 & \\
\hline & & Peripsocus phaeopterus (Stephens 1836) & \\
\hline & & Peripsocus subfasciatus (Rambur 1842) & \\
\hline & Psocidae & Blaste conspurcata (Rambur 1842) & Kalinović et al., 1979 \\
\hline & & Metylophorus nebulosus (Stephens 1836) & $\begin{array}{l}\text { Kalinović et al., 1980; } \\
\text { Mihaljević et al.,1999 }\end{array}$ \\
\hline & & Psococerastis gibbosa (Sulzer 1776) & \\
\hline & & Psocus bipunctatus (Linnaeus 1761) & Kalinović et al., 1979 \\
\hline & & Trichadenotecnum majus (Kolbe 1880) & $\begin{array}{l}\text { Kalinović et al., 1980; } \\
\text { Mihaljević et al., } 1999\end{array}$ \\
\hline & & Trichadenotecnum sexpunctatum (Linnaeus & \\
\hline & & 1758) & \\
\hline & Stenopsocidae & Stenopsocus immaculatus (Stephens 1836) & \\
\hline & & Stenopsocus stigmaticus (Imhoff \& Labram & \\
\hline & & 1842) & \\
\hline \multirow{8}{*}{$\begin{array}{l}\text { Suborder } \\
\text { Troctomorpha }\end{array}$} & Family & Species & Source of data \\
\hline & Liposcelididae & Liposcelis bostrychophila Badonnel 1931 & $\begin{array}{l}\text { Kalinović et al., 1980; } \\
\text { Mihaljević et al., } 1999\end{array}$ \\
\hline & & Liposcelis decolor (Pearman 1925) & \\
\hline & & Liposcelis entomophila (Enderlein 1907) & \\
\hline & & Liposcelis keleri Gunther 1974 & Kalinović et al., 1977 \\
\hline & & Liposcelis palatina Roesler 1954 & Kalinović et al., 1980 \\
\hline & & Liposcelis pearmani Lienhard 1990 & \\
\hline & & Liposcelis silvarum (Kolbe 1888) & \\
\hline
\end{tabular}

Legend: • old names (synonyms) 


\section{Acknowledgements}

I am thankful to Lawrence J. Hribar PhD (Florida Keys Mosquito Control District, Marathon, Florida, USA) for all improvements in this manuscript. Also, I would like to give my thanks to all entomologists for determinations and reviews of collected insects.

\section{References}

Ábrahám, L., 2008. "Baseline Survey and Biological Monitoring of Butterflies (Lepidoptera) in Croatian Areas Along the Drava River, 237-248". In: Biodiversity studies along the Drava river (Ed. J. J. Purger). University of Pécs, Pécs, $248 \mathrm{pp}$.

Adamović, Ž. R., 1975. A preliminary survey of the mosquitoes (Diptera: Culicidae) of Obedska Bara, Serbia. Bulletin of the Natural History Museum in Belgrade Serie B 30 (1): 75-80.

Bistrović, M., 2012. Comparisson of Sampling Methods of Aquatic Insects (Heteroptera,Coleoptera) in the Nature Park Kopački rit. MSc. thesis, J J Strossmayer University of Osijek, Osijek, Croatia, 66 pp. (in Croatian with English abstract).

Bogdanović, T., P. Durbešić \& J. Mikuska, 2002. "Dragonfly Fauna of the Kopački Rit Wetlands, 741-749. In: Limnological Reports. 34th International Association of Danube Research Conference (Eds. G. Brezeanu \& R. Ştiucă). Editura Academiei Romăne, Bucharest, 895 pp.

Bognar, A., 1990. Geomorfologija baranje. Savez geografskih društava Hrvatske. Zagreb, 312 pp.

Brigić, A., S. Vujčić-Karlo \& Z. Stančić, 2003. Carabidae Fauna in different terrestrial habitats of Krapje Đol. Nature Park Bulletin 5 (1/2): 13-26.

Čerba, D., 2010. Functional Role of Chironomid Larvae (Chironomidae, Diptera) in Submerged Macrophyte Communities of Sakadaš Lake. Ph.D. thesis, University of Zagreb, Zagreb Croatia, 156 pp. (in Croatian with English abstract).

Čurčić, S. \& M. Stanković, 2011. The ground beetles (Coleoptera:Carabidae) of the Zasavica Special Nature Reserve (Serbia). Acta Entomologica Serbica 16 (1/2): 61-79.

Domić, D., 2009. Sezonska Dinamika Trčaka (Carabidae, Coleoptera) u Kopačkom Ritu s Posebnim Osvrtom na Vrste Bioindikatore Zagađenja. MSc. thesis, J J Strossmayer University of Osijek, Osijek, Croatia, 108 pp. (in Croatian with English abstract).

Draganić, R., 2000. Characteristics of the Noctuid Fauna (Lepidoptera: Noctuidae) of Kopački rit. MSc. thesis, J J Strossmayer University of Osijek, Osijek, Croatia, 37 pp. (in Croatian with English abstract).

Durbešić, P., S. Vujčić-Karlo \& L. Šerić, 2000. "The Ground Beetles (Coleoptera, Carabidae) of Some of the Forest Communities of Lonjsko Polje, 151-156" In: Limnological Reports. 33th International Association of Danube Research Conference (Ed. J. Horvatić). Grafika, Osijek, 547 pp.

Fauna Europaea, 2013. Fauna Europaea. (Web page: http://www.faunaeur.org) (Date accessed: 21 December 2013).

Hill, B. T. \& B. Beinlich, 2000. The dragonfly community of a communal cattle pasture in the Sava floodplain (Croatia) with special reference to the biology of Lestes barbarus (Fabricius, 1798) (Zygoptera: Lestidae). Exuviae 7 (1): 1-16.

Horvatić, J., 2002. Park Prirode Kopački rit - Plan Upravljanja Parkom Prirode Kopački Rit. Sektorska studija Biodiverzitet. Ministarstvo zaštite okoliša i prostornog uređenja. Zagreb, 344 pp (in Croatian with English abstract).

IUCN, 1994. IUCN Red list categories. Prepared by the IUCN Species Survival Commission. (Web page: http://www.iucnredlist.org) (Date accessed: 4 February 2014).

IUCN, 2014. IUCN Red list of threatened species. Version 2014.1. (Web page: http://www.iucnredlist.org) (Date accessed: 26 June 2014). 
Jeličić, Ž., A. Vujić, G. Vignjević \& E. Merdić, 2010. Hoverflies (Diptera: Syrphidae) of Kopački rit Nature Park, NE Croatia. Entomologia Croatica 14 (3/4): 7-18.

Kalinović, I., G. Pivar \& K. K. Günther, 1977. Psocoptera-Fauna im freiland slawoniens und baranyas (Hemipteroidea). Acta Entomologica Jugoslavica 13 (1/2): 51-58.

Kalinović, I., K.K. Günther \& G. Pivar, 1979. Psocoptera fauna on territory Kopački rit. Zbornik radova Poljoprivrednog fakulteta u Osijeku 5 (1): 51-65 (in Croatian with English abstract).

Kalinović, I., G. Pivar \& K. K. Günther, 1980. Contribution to the investigation of Psocoptera in the special zoological reservation Kopački rit in Baranya. Acta Entomologica Jugoslavica, 16 (1/2): 111-119.

Kalkman,V. J., J. P. Boudot, R. Bernard, K. J. Conze, G. De Knijf, E. Dyatlova, S. Ferreira, M. Jović, J. Ott, E. Riservato \& G. Sahlén, 2010. European Red List of Dragonflies. Publication Office of the European Union, Luxembourg, $30 \mathrm{pp}$.

Kovačević, T., 2011. Compostion and Abundance of Chironomid Larvae (Chironomidae,Diptera) in Periphyton Community on Artifitial Substrates. MSc thesis, J J Strossmayer University of Osijek, Osijek, Croatia, 73 pp. (in Croatian with English abstract).

Krčmar, S., 2005. Seasonal abundance of horse flies (Diptera: Tabanidae) from two locations in Eastern Croatia. Journal of Vector Ecology 30 (2): 316-321.

Krčmar, S., E. Merdić \& S. Vidović, 1996. Butterflies of Baranya (Lepidoptera, Rhopalocera) contribution to knowledge of butterflies of Croatia. Poljoprivreda 2 (1/2): 33-40. (in Croatian with English abstract).

Krčmar, S., E. Merdić \& M. Kopi, 2005. Diurnal periodicity in the biting activity of horsefly species in the Kopački rit Nature Park, Croatia (Diptera: Tabanidae). Entomologia Generalis 28 (2): 139-146.

Krčmar, S., J. Mikuska \& P. Durbešić, 2006 a. Ecological characteristics of certain species of horse flies (Diptera: Tabanidae) in Kopački rit Nature Park, Croatia. Periodicum biologorum 108 (1): 11-14.

Krčmar S., A. Mikuska \& J. Majer, 2006 b. Ecological notes on horse flies of some flooded areas in the middle course of the Drava river (Diptera: Tabanidae). Entomologia Generalis, 28 (4): 275-282.

Krčmar, S., A. Mikuška \& M. Jasika, 2009. Horsefly fauna of three different forest communities in the Danube river Floodplain in Croatia (Diptera: Tabanidae). Entomologia Generalis 32 (1): 23-34.

Kulundžić, K., 2011. Faunal Characteristics of Scarab Beetles (Scarabaeidae, Coleoptera) in the Kopački rit Nature Park. MSc thesis, J J Strossmayer University of Osijek, Osijek, Croatia, 63 pp (in Croatian with English abstract).

Kurbalija, A., 2012. Review Entomofauna of Wetland Habitats of International Importance in the Republic of Croatia. MSc thesis, J J Strossmayer University of Osijek, Osijek, Croatia, 128 pp. (in Croatian with English abstract).

Maltchik, L., M. Schmidt-Dalzochio, C. Stenert \& A. S. Rolon, 2012. Diversity and distribution of aquatic insects in Southern Brasil wetlands: implications for biodiversity conservation in a Neotropical region. Revista de Biologia Tropical 60 (1): 273-289.

Merdić, E., 1993. Mosquitoes (Diptera, Culicidae) of the special zoological reserve Kopački rit (NE Croatia). Natura Croatica 2 (1): 47-54.

Merdić, E. \& M. Sudarić, 2003. Effects of prolonged high water level on the mosquito fauna in Kopački rit Nature Park. Periodicum biologorum 105 (2): 189-193.

Merdić, E., N. Keža \& Z. Csabai, 2005. Aquatic insects in Kopački rit Nature Park (Heteroptera: Nepomorpha, Gerromorpha and Coleoptera: Hydradephaga, Hydrophiloidea). Natura Croatica 14 (4): 263-272.

Merdić, E., S. Krčmar, M. Sudarić-Bogojević \& Ž. Jeličić, 2007. Response of mosquitoes to different synthetic and natural olfactory attractants (Diptera: Culicidae). Entomologia Generalis 30 (4): 253-261.

Merdić, E., Ž. Jeličić, M. Kovačević, V. Leko, M. Sudarić-Bogojević, I. Boca \& Ž. Zahirović, 2008. Distribution of the Annulipes group (Diptera: Culicidae) of Eastern Croatia. Entomologia Croatica 12 (2): 9-22.

Mihaljević, M., D. Getz, Z. Tadić, B. Živanović, D. Gucunski, J. Topić, I. Kalinović \& J. Mikuska, 1999. Kopački rit Research Survey and Bibliography. Hazu, Zavod za Znanstveni rad u Osijeku, Osijek, 188 pp. (in Croatian with English, German and Hungarian abstract).

Mitsch, W. J. \& J. G. Gosselink, 2000. Wetlands. John Wiley \& Sons Inc. New York, 920 pp. 
Nedeljković, Z., A. Vujić, A. Ricarte, S. Radenković \& S. Šimić, 2010. New data on the genus Syrphus Fabricius, 1775 (Diptera: Syrphidae) from the Balkan peninsula including the first record of Syrphus nitidifrons Becker, 1921. Acta Entomologica Serbica 15 (1): 91-105.

Nieto, A. \& K. N. A. Alexander, 2010. European Red List of Saproxylic Beetles. Publications office of the European Union. Luxembourg, $46 \mathrm{pp}$.

Perović, F., E. Merdić \& G. Perović, 2006. Sawflies (Hymenoptera, Symphyta) in the biotopes of Kopački rit. Natura Croatica 15 (4): 189-201.

Pešić, S., 2012. New weevils (Coleoptera: Curculionoidea) in the special nature reserve Zasavica. Acta Entomologica Serbica 17 (1/2): 123-134.

Pil, N. \& M. Stanković, 2006. Cerambycidae (Coleoptera) of the Zasavica Special Nature Reserve (Serbia). Acta Entomologica Serbica 11 (1/2): 33-43.

Price, P. W., 1997. Insect Ecology. John Wiley \& Sons Inc. New York, 874 pp.

Pulitika, T., 2000. Characteristics of the Fauna of Moths in Kopački rit. MSc Thesis, J J Strossmayer University of Osijek, Osijek, Croatia, 29 pp (in Croatian with English abstract).

Ramsar Convention - The ramsar list. (Web page: http://www.ramsar.org) (Date accessed 25 June 2013).

Radenković, S. R., A. V. Vujić, \& S. D. Šimić, 2004. New data on hoverfly diversity (Insecta: Diptera: Syrphidae) of the special nature reserve the Obedska Bara marsh (ramsar site in Serbia). Proceedings for Natural Sciences Matica Srpska Novi Sad 107 (1): 21-31.

Reinstra, E., 2004. Dagvlinders in Kopački rit. Vlinders 2 (1): 10-13.

Schneider-Jacoby, M., 1994. Sava and Drava - ecological value and future of the two main rivers in Croatia. Periodicum biologorum 96 (4): 348-356.

Schneider-Jacoby, M. \& H. Ern, 1993. Lonjsko Polje Nature Park. Croatian Ecological Society. Zagreb, 135 pp.

Springer, O. P., M. Mihaljević, D. Getz, S. Božičević, A. Bognar, J. Topić, E. Merdić \& S. Krčmar, 2003. Kopački rit Park Prirode. Ministarstvo Zaštite Okoliša i Prostornog Uređenja RH. Zagreb, 155 pp.

Stanković, M., 2010. Specijski diverzitet specijalnog rezervata prirode Zasavica. Ecologica 17 (58): 167-172.

Stanković, M., 2011. "Strictly protected and endangered species in fauna of the Special Nature Reserve Zasavica, 45-46." XXII Symposium Internationale Entomofaunisticum Europae Centralis (29 June - 3 July 2011, Varaždin, Croatia), $71 \mathrm{pp}$.

State Institute for Nature Protection, 2014. (Web page: http://www.dzzp.hr) (Date accessed 17 January 2014).

Sudarić-Bogojević, M., A. Ignjatović-Ćupina, D. Petrić \& E. Merdić, 2009. "Preliminarna istraživanja braničevki (Diptera: Simulidae) u Parku prirode Kopački rit 210-211". 10th Croatian Biological Congress with International Participation (14 - 20 September 2009, Osijek, Croatia), 250 pp.

Szent-Ivány, J., 1944. Lepidopterenfaunistische und ökologische beobachtungen in der umgebung der erzherzog Albrecht biologischen station des Ungarischen national muzeums. Albertina 1 (1): 135-148.

Tallósi, B., 2008. "Population-Level Baseline Surveying and Preparative Investigations for the Monitoring of Carabid Beetles (Coleoptera, Carabidae) in Areas Along the Drava River and in Baranja (Croatia), 165-220". In: Biodiversity studies along the Drava river (Ed. J. J. Purger). University of Pécs, Pécs, 248 pp.

Temunović, M., L. Šerić-Jelaska \& P. Durbešić, 2007. Diversity of water beetles (Hydradephaga, Coleoptera) in temporary ponds of Lonjsko polje Nature Park, Croatia. Entomologia Croatica 11 (1/2): 13-24.

Trilar, T. \& S. Krčmar, 2005. Contribution to the knowledge of louse flies of Croatia (Diptera: Hippoboscidae). Natura Croatica 14 (2): 131-140.

Turić, N., 2007. Aquatic Insects (Heteroptera, Coleoptera) in Kopački rit Nature Park with Special Reference to Rare, Endangered and Protected Species. MSc thesis, J J Strossmayer University of Osijek, Osijek, Croatia, 105 pp. (in Croatian with English abstract).

Turić, N., E. Merdić, B. K. Hackenberger, Ž. Jeličić, G. Vignjević \& Z. Csabai, 2012. Structure of aquatic assemblages of Coleoptera and Heteroptera in relation to habitat type and flood dynamic structure. Aquatic Insects 34 (1): 189-205. 
Van Swaay, C., A. Cuttelod, S. Collins, D. Maes, M. López Munguira, M. Šašić, J. Settele,R. Verovnik, T. Verstrael, M. Warren, M. Wiemers \& I. Wynhof, 2010. European Red List of Butterflies. Publications Office of the European Union. Luxembourg, $48 \mathrm{pp}$.

Vignjević, G., Ž. Zahirović, N. Turić \& E. Merdić, 2010. Moths (Lepidoptera: Heterocera) of Kopački rit Nature park results of preliminary research. Entomologia Croatica 14 (3/4): 17-32.

Vrućina, I., 2010. Influence of Natural and Artifical Light on Mosquito Behavior and Activities (Diptera, Culicidae) in Urban and Forest Area of Eastern Croatia. MSc thesis, J J Strossmayer University of Osijek, Osijek, Croatia, 122 pp. (in Croatian with English abstract). 